On the Relation Between the Velocity Coefficient and Boundary Value for Solutions of the One-dimensional Wave Equation

R. Michael Lewis

and

William W. Symes

May, 1990

(revised April 1991)

TR90-12 



\title{
ON THE RELATION BETWEEN THE VELOCITY COEFFICIENT AND BOUNDARY VALUE FOR SOLUTIONS OF THE ONE-DIMENSIONAL WAVE EQUATION *
}

\author{
R. MICHAEL LEWIS AND WILLIAM W. SYMES †
}

\begin{abstract}
The one-dimensional acoustic wave equation is a simple model of wave propagation in layered media. As such, it is used in theoretical seismology to study the relation between sound velocity and the surface response or seismogram (boundary value) as a simple instance of the reflection seismology problem.

Questions about the nature of the dependence of boundary values on the velocity coefficient in the wave equation arise naturally in this context, particularly in connection with perturbational techniques. We study the dependence on the velocity coefficient of boundary values for solutions of the onedimensional wave equation. We present estimates for both the Lipschitz continuity and the linearization error for the map between velocity coefficient and boundary value. In particular, we show that this relation is Lipschitz continuous for velocities in $H^{2}$ and differentiable for velocities in $H^{3}$. We also discuss the anomalous smoothness of this map in "oscillatory" directions, which helps explain a key idea in reflection seismology.
\end{abstract}

Key Words. wave propagation, one-dimensional acoustics, inverse problems, stability

AMS(MOS) subject classification. Primary 35L05, secondary 35R30, 73D99, 86A15

1. Introduction. Various wave propagation problems in a plane-stratified halfspace $\{z>0\}$ can be modeled by the one-dimensional acoustic wave equation:

$$
\frac{1}{c^{2}(z)} \partial_{t t}^{2} u-\partial_{z z}^{2} u=0
$$

In this model the mechanical properties of the medium are described by the sound velocity $c(z)$.

We impose the boundary and initial conditions

$$
\begin{aligned}
\partial_{z} u(0, t) & =f(t) \\
u(z, t) & \equiv 0 \quad t<0,
\end{aligned}
$$

where the Neumann datum $f$ vanishes for $|t|$ large so that the disturbance is transient. The velocity coefficient $c$ is a strictly positive function and for the moment is assumed to be $C^{\infty}$.

If the datum $f$ is $C^{\infty}$ then we can sensibly define a map $F_{f}: C^{\infty} \rightarrow C^{\infty}$ by

$$
F_{f}: c \mapsto \partial_{t} u(0, t)
$$

If $u$ represents displacement, then the map $F_{f}$ relates the velocity coefficient and the surface velocity. In the context of the reflection seismology method in oil exploration,

* Research sponsored by AFOSR-89-0363. A version of this work appeared in R. M. Lewis' doctoral thesis, written under the supervision of W. W. Symes in the Department of Mathematical Sciences, Rice University.

$\dagger$ Department of Mathematical Sciences, Rice University, Houston, Texas, 77251-1892. 
$F_{f}$ represents the relation between the surface observations ("seismogram") and the unknown subterranean material properties.

The conditions (1), (2), and (3) form a well-posed initial-boundary-value problem in the half-space $\{z>0\}$ for the distribution datum $f=-\delta$ since the velocity coefficient is sufficiently smooth. However, in this case we qualify the definition of the map between velocity and boundary value given by (4) to account for the absence of smoothness of $f=-\delta$. The progressing wave expansion for $u$ (see Section 2.3) shows that $\partial_{t} u$ has a well-defined trace

$$
\partial_{t} u(0, t)=\text { constant } \cdot \delta(t)+v(t)
$$

where $v \in C^{\infty}[0, T]$ for any $T>0$. We define the map $F$ to be

$$
F: c \mapsto v(t)
$$

Since for smoother choices of $f$ we have

$$
F_{f}(c)=f *(\text { constant } \cdot \delta+F(c))
$$

properties for $F_{f}$ follow from those of $F$ via well-understood properties of convolution. Accordingly, we restrict attention to the case $f=-\delta$, the so-called impulse response problem.

To this point we have assumed that the velocity coefficient $c$ is $C^{\infty}$. Our goal in this paper is to establish weaker regularity conditions on $c$ under which $F$ extends to a continuous map and a differentiable map. Our main results are that

(a) $F$ is Lipschitz continuous as a map $H^{2} \rightarrow L^{2}$, and

(b) $F$ is differentiable as a map $H^{3} \rightarrow L^{2}$ and the derivative is Lipschitz continuous, as we shall now describe.

Let $u$ be the solution of

$$
\begin{aligned}
\frac{1}{c^{2}(z)} \partial_{t t}^{2} u-\partial_{z z}^{2} u & =0, \quad z>0 \\
\partial_{z} u(0, t) & =-\delta(t) \\
u & \equiv 0, \quad t<0 .
\end{aligned}
$$

A consequence of the estimates we will present in Section 2.5 is that the map $F$ relating sound velocity and boundary value via (5) extends to a map $H^{1} \rightarrow L^{2}$.

Our first major result is that $F$ extends to a Lipschitz continuous map from $H^{2}$ to $L^{2}$.

THEOREM 1.1. Given a velocity $c$ and $T>0$, there exist $D$ and $r>0$ such that if $\delta c \in H^{2}[0, D],\|\delta c\|_{H^{2}[0, D]}<r$, then

$$
\|F(c+\delta c)-F(c)\|_{L^{2}[0, T]} \leq K\|\delta c\|_{H^{1}[0, D]} .
$$

The constant $K$ depends on the depth $D,\|c\|_{H^{2}[0, D]}$,

$$
c_{\min }=\inf _{0 \leq z \leq D} c(z)
$$


and $r$.

Our second theorem concerns the differentiability of $F$. Formally, the first-order effect of changing the velocity from $c$ to $c+\delta c$ is to perturb the solution $u$ by $\delta u$, where $\delta u$ is the solution of the linearized problem

$$
\begin{aligned}
\frac{1}{c^{2}(z)} \partial_{t t}^{2} \delta u-\partial_{z z}^{2} \delta u & =\frac{2 \delta c(z)}{c^{3}(z)} \partial_{t t}^{2} u, & & z>0 \\
\partial_{z} \delta u(0, t) & =0 & & \\
\delta u & \equiv 0, & & t<0 .
\end{aligned}
$$

As with the trace $\partial_{t} u(0, t)$ there is a highly singular part and a smooth part to $\partial_{t} \delta u(0, t)$. The progressing wave expansion for $\delta u$ shows that

$$
\partial_{t} u(0, t)=\text { constant } \cdot \delta^{\prime}(t)+\text { constant } \cdot \delta(t)+v(t),
$$

We will consider a formal linearization $D F$ of $F$ defined by

$$
D F[c]: \delta c \mapsto v(0, t)
$$

In order to determine whether $D F$ really is the derivative of $F$, we will estimate the Taylor's series remainder

$$
F(c+\delta c)-F(c)-D F[c](\delta c)
$$

for all velocity perturbations $\delta c$ which are "sufficiently small."

Our second result - the more significant for applications - is that $F$ is differentiable as a map $H^{3} \rightarrow L^{2}$, and the derivative is Lipschitz continuous.

THEOREM 1.2. Given a velocity $c$ and $T>0$, there exists $D$ such that $F$ is differentiable at $c$ as a map $F: H^{3}[0, D] \rightarrow L^{2}[0, T]$, and the derivative is Lipschitz continuous.

More precisely, given $c$ and $T>0$, there exist $D$ and $r>0$ such that:

(i) For all $\delta c \in H^{2}[0, D],\|\delta c\|_{H^{2}[0, D]}<r$, the linearization error satisfies

$$
\|F(c+\delta c)-F(c)-D F[c](\delta c)\|_{L^{2}[0, T]} \leq K\|\delta c\|_{L^{2}[0, D]}\|\delta c\|_{H^{2}[0, D]}+B\|\delta c\|_{L^{2}[0, D]}^{2} .
$$

The constant $K$ depends on the depth $D,\|c\|_{H^{2}[0, D]}$,

$$
c_{\min }=\inf _{0 \leq z \leq D} c(z)
$$

and $r$, while the constant $B$ depends on the depth $D,\|c\|_{H^{3}[0, D]}, c_{\min }$, and $r$. then

(ii) The derivative depends continuously on $c$ in the sense that if $\|\tilde{c}-c\|_{H^{2}[0, D]}<r$,

$$
\|D F[\tilde{c}]-D F[c]\| \leq B\|\tilde{c}-c\|_{H^{2}[0, D]}<r
$$

where the constant $B$ depends on the depth $D,\|c\|_{H^{3}[0, D]}, c_{m i n}$, and $r$, and the operator norm is taken over operators from $H^{2}[0, D]$ to $L^{2}[0, T]$. 
The appearance of $\|c\|_{H^{3}[0, D]}$ in the regularity estimates suggests that $F$ is differentiable with Lipschitz continuous derivative only as a map $H^{3}[0, D] \rightarrow L^{2}[0, T]$. The smoothness restriction $c \in H^{3}$ is a consequence of the singular nature of the boundary datum $f=-\delta$. For smoother $f$ we can relax the smoothness requirement on the velocity at the expense of the appearance of derivatives of $f$ in our estimates. For such a resuit, see [1].

The particular form of the linearization error estimate has further significance. On the one hand, the error estimate indicates that linearization is valid only for velocity perturbations with small $H^{2}$-norm. On the other hand, it turns out that we can be much more optimistic about the accuracy of the linearization of $F$ in the special case where $\delta c$ is highly oscillatory. The key point is that the map $F$ is very smooth in these directions, as we shall now discuss. This observation is significant for the use of the problem (6) as a model in reflection seismology and helps explain an item of the conventional wisdom in that subject.

To simplify notation, let $\|\cdot\|_{k}$ denote the norm on $H^{k}[0, D]$, with $H^{0}[0, D]=$ $L^{2}[0, D]$. Suppose that the support of $\delta c$ is contained in $(0, D)$. We will say that $\delta c$ is oscillatory if

$$
\left\|\int_{0}^{z} \delta c\right\|_{0} \ll\|\delta c\|_{0}
$$

For convenience we will write

$$
\left\|\int_{0}^{z} \delta c\right\|_{0}=\varepsilon\|\delta c\|_{0}
$$

where $\varepsilon$ should be thought of as a small number. For our discussion, we will also assume that the $H^{3}$-norm of $\delta c$ satisfies $\|\delta c\|_{3} \leq R$.

If $\delta c$ is oscillatory, then

$$
\|\delta c\|_{0}^{2}=\int_{0}^{D} d z \delta c^{\prime}(z)\left(\int_{0}^{z} \delta c\right) \leq\left\|\int_{0}^{z} \delta c\right\|_{0}\|\delta c\|_{1} \leq \varepsilon\|\delta c\|_{0}\|\delta c\|_{1}
$$

and consequently

$$
\|\delta c\|_{0} \leq \varepsilon\|\delta c\|_{1} .
$$

Thus if $\delta c$ is oscillatory we have

$$
\begin{aligned}
\|\delta c\|_{0}\|\delta c\|_{2} & \leq\|\delta c\|_{0}\left(\|\delta c\|_{1}+\left\|\delta c^{\prime \prime}\right\|_{0}\right) \\
& \leq\|\delta c\|_{0}\left(\|\delta c\|_{1}+\left\|\delta c^{\prime}\right\|_{0}^{\frac{1}{2}}\left\|\delta c^{\prime \prime \prime}\right\|_{0}^{\frac{1}{2}}\right) \\
& \leq\|\delta c\|_{0}\|\delta c\|_{1}^{\frac{1}{2}}\|\delta c\|_{3}^{\frac{1}{2}} \\
& \leq \varepsilon R^{\frac{1}{2}}\|\delta c\|_{1}^{\frac{3}{2}} .
\end{aligned}
$$

In this case, the estimate of the linearization error in Theorem 1.2 reduces to

$$
\|F(c+\delta c)-F(c)-D F[c](\delta c)\|_{L^{2}[0, T]} \leq C \varepsilon R^{\frac{1}{2}}\|\delta c\|_{1}^{\frac{3}{2}} .
$$


The point is that the error estimate is superlinear in the $H^{\mathbf{1}}$-norm of $\delta c$ as long as $\delta c$ is oscillatory. Moreover, the oscillation of $\delta c$ enables us to control the size of the term $C \varepsilon R^{\frac{1}{2}}$.

As an example of a family of oscillatory perturbations, let $\chi \in C_{0}^{\infty}(0, D), 0 \leq \chi \leq 1$, and $\chi \equiv 1$ on some interval $\left[z_{0}, z_{1}\right] \subset(0, D)$. For $\varepsilon>0$ and $\alpha \geq 1$ define

$$
\delta c_{\varepsilon}(z)=\varepsilon^{\alpha} \chi(z) \sin \frac{z}{\varepsilon}
$$

Then

$$
\left\|\int_{0}^{z} \delta c_{\varepsilon}\right\|_{0}=O(\varepsilon)\left\|\delta c_{\varepsilon}\right\|_{0}
$$

while

$$
\left\|\delta c_{\varepsilon}\right\|_{k}=O\left(\varepsilon^{\alpha-k}\right)
$$

In particular, if $\alpha \geq 1$ then $\delta c_{\varepsilon} \in H^{1}$, and if $\alpha>1$ then $\left\|\delta c_{\varepsilon}\right\|_{1} \rightarrow 0$ as $\varepsilon \rightarrow 0$.

If $\alpha=3, \delta c_{\varepsilon}$ stays in a bounded set in $H^{3}$ as $\varepsilon \rightarrow 0$. On the other hand, for $1<\alpha<3,\left\|\delta c_{\varepsilon}\right\|_{3} \rightarrow \infty$ as $\varepsilon \rightarrow 0$. Nonetheless, since $\delta c_{\varepsilon} \in H^{1}, F\left(c+\delta c_{\varepsilon}\right)$ is still defined and we have the estimate

$$
\|F(c+\delta c)-F(c)-D F[c](\delta c)\|_{L^{2}[0, T]} \leq C \varepsilon^{\frac{\alpha-1}{2}}\|\delta c\|_{1}^{\frac{3}{2}}
$$

Since $\alpha>1$, the quantity $C \varepsilon^{\frac{\alpha-1}{2}} \rightarrow 0$ with $\varepsilon$. Thus the linearization error is small, even though the $H^{3}$-norm (and the $H^{2}$-norm, for $1<\alpha<2$ ) of $\delta c_{\varepsilon}$ is unbounded as $\varepsilon \rightarrow 0$ ! This example illustrates the anomalous smoothness of $F$ in the directions of oscillatory perturbations.

This observation helps to make precise an idea central to the mathematical methods of reflection seismology. One of the tenets of reflection seismology methods in petroleum exploration is that linearization of the map $F$ (or its equivalent in more complex models) is valid as a means of studying the effects of oscillatory perturbations of a smooth reference medium. This technique is the basis of many of the methods used to analyze reflection data in oil exploration. The preceding analysis of the linearization error in the case of oscillatory perturbations supports this belief, and gives a rigorous justification for the "velocity/reflectivity" dichotomy that makes seismic data interpretation possible.

Another important property of the map $F$ is the effect of slowly-varying perturbations, the obverse of the case we just considered. The effect of slowly-varying perturbaticns is related to the smoothness restriction $c \in H^{3}$ required for differentiability. To understand how $F$ reacts to slowly-varying perturbations we will consider the closely related impedance problem. The issue of regularity for the impedance problem was studied by Symes in [4]. There he considered the relation between coefficient and boundary value for Webster's horn equation:

$$
\begin{array}{rlrl}
\left(\eta(x) \partial_{t t}^{2}-\partial_{x}\left(\eta(x) \partial_{x}\right)\right) v & =0, & & x>0 \\
\partial_{x} v(0, t) & =-\delta(t) & \\
v & \equiv 0, \quad t<0 .
\end{array}
$$


The quantity $\eta(x)$ is the impedance. If we set $\sigma(x)=\partial_{x} \log \eta(x)$, then we obtain the more convenient formulation

$$
\begin{array}{rlrl}
\left(\partial_{t t}^{2}-\partial_{x x}^{2}-\sigma(x) \partial_{x}\right) v & =0, & & x>0 \\
\partial_{x} v(0, t) & =-\delta(t) & \\
v & \equiv 0, \quad t<0 .
\end{array}
$$

In this case the coefficient $\sigma$ and and the boundary value $\partial_{t} v(0, t)$ are related by the map $\tau$ :

$$
\tau: \sigma \mapsto \partial_{t} v(0, t)
$$

Symes showed that $\tau$ is a $C^{1}$-diffeomorphism from $L^{2}[0, T]$ to $L^{2}[0, T]$; i.e., the relation is differentiable for impedances in $H^{1}$.

The velocity problem (6) can be transformed into the impedance problem via the change of variable

$$
x=\int_{0}^{z} d \zeta \frac{1}{c(\zeta)}
$$

The impedance is given by

$$
\eta(x)=c^{-1}(z(x))
$$

This change of variable is called the travel-time transformation. The quantity $x$ in (9) is called the travel-time, and is the time it takes a signal traveling downward from the surface to arrive at depth $z$.

A salient difference between the velocity problem and the impedance problem is the effect of the travel-time transformation in "straightening out" the characteristic curves associated with the differential operators. In the impedance problem (8) the characteristics are independent of $\sigma$. In the velocity problem (6) different velocities give rise to different characteristics, a feature that enormously complicates the proof of the reguiarity theorem and forces us to require additional smoothness on the velocity $c$ in order that $F$ be differentiable. This simplifying effect of the travel-time transformation (9) reflects a fundamental feature of the map $F$.

As previously noted, Symes established differentiability on $H^{1}$ for the impedance problem. By contrast, for the velocity problem a linearization estimate in terms of the $H^{1}$-norm of $\delta c$ was derived only for oscillatory perturbations of a smooth $\left(H^{3}\right)$ velocity. That no better result is possible is inherent to the velocity problem. While the map $F$ is surprisingly well-behaved for oscillatory perturbations in the velocity, it is extremely sensitive to slowly-varying perturbations in the velocity.

The extreme sensitivity of $F$ to changes in the slowly-varying component of the velocity is manifest in a phenomenon known as the travel-time shift. The travel-time shift refers to the possibly catastrophic effect of a small change in the sound velocity. The arrival times of reflected signals at the surface are determined by the sound velocity in the medium. On the one hand, a small, slowly-varying perturbation in the sound 
velocity will produce only small changes in arrival times. However, if the reflected signals are highly oscillatory this can lead to a dramatic change in the surface measurements, since the highly oscillatory features of the signal will have undergone a shift relative to their former locations. The additional smoothness requirements of our regularity theorem serve to ameliorate this unfortunate behavior. For a fuller discussion of the travel-time instability, see [3] and the references cited there.

The remainder of this paper is devoted to the proof of Theorems 1.1 and 1.2. The proof is rather lengthy. It is perhaps possible that a shorter proof might be possible via a study of the travel-time map. However, such an argument would be inextricably bound to the result for the impedance problem, which has no analog for problems with multiple wave speeds and most especially for the multi-dimensional wave equation. This consideration motivates the proof given here as a pattern of attack on the multidimensional problem using various energy estimates.

The proof extends the methods employed by Symes in his study of Webster's horn equation in [4], namely, sideways energy estimates and the progressing wave expansion. Sections 2.2 and 2.3 introduce these ideas. The application of these techniques to the velocity problem (6) is complicated by the more complex characteristic geometry of the velocity problem. The heart of the proof lies in Section 2.5, where we use the sideways energy identity to derive trace estimates for solutions of the wave equation. These estimates are sharp propagation of regularity results. In Sections 2.6 and 2.7 we use these trace estimates to compare solutions of the wave equation associated with different velocities. The remaining sections complete the proof of the theorems.

\section{Preliminaries to the proofs of Theorems 1.1 and 1.2 .}

2.1. Time-depth relationships. In this section we will we will define the depth $D$ and distance $r$ which appear in Theorems 1.1 and 1.2 and discuss the relationship between the time and depth domains. We will assume that $F(c), F(c+\delta c)$, and $D F[c](\delta c)$ are given for time $t$ in an interval $[0, T]$. The definition of a related interval in depth $z$ is somewhat complicated - here we encounter the first of many difficulties caused by the difference in the characteristics of the wave operators associated with the different velocities.

Given $c$, let $c_{1}=c$ and $c_{2}=c+\delta c$, and for $i=1,2$, define the two travel-times

$$
\phi_{i}(z)=\int_{0}^{z} d \zeta \frac{1}{c_{i}(\zeta)}
$$

Since the $\phi_{i}$ are increasing functions, they have well-defined inverses.

Given a time $T$, define the depth $d$ by

$$
d=\phi_{1}^{-1}\left(\frac{T}{2}\right) .
$$

This defines a "domain of dependence" for the surface measurements on the interval $[0, T]$. If signals travel with velocity $c_{1}$, then $d$ is the deepest point a signal can reach and still return to the surface before time $T$. 
Now choose $b>0$ and set

$$
D=\phi_{1}^{-1}\left(\frac{T}{2}+\frac{b}{2}\right)
$$

With $b$ and $D$ thus defined, suppose that $\left\|c_{2}-c_{1}\right\|_{H^{1}[0, D]}$ is small enough that

$$
\left\|\phi_{2}-\phi_{1}\right\|_{L^{\infty}[0, D]}<\frac{b}{2}
$$

Then with these choices we have

$$
\begin{aligned}
\tau+\phi_{1}(D) & \geq T-\phi_{1}(D) \\
\tau+\phi_{2}(D) & \geq T-\phi_{1}(D)
\end{aligned}
$$

for all $\tau \geq-b / 2$.

The depth $D$ is slightly deeper than the depth reached at time $T / 2$ by signals travelling at speed $c_{1}$. The slightly enlarged depth domain is required in order to compare signals travelling at a slightly slower velocity $c_{2}$.

Now we can say how small $\delta c$ must be in order for the estimates in Theorems 1.1 and 19 to hold. Let $c_{\min }$ and $c_{\max }$ be lower and upper bounds on the velocity $c_{1}$ on the interval $[0, D]$ : for all $z \in[0, D]$

$$
0<c_{\min } \leq c_{1}(z) \leq c_{\max }
$$

We will require that $\delta c$ be "sufficiently" small in $H^{2}$ : We choose $r>0$ small enough that if $\left\|c_{2}-c_{1}\right\|_{H^{2}[0, D]}<r$, then

$$
\begin{gathered}
\left\|\phi_{2}-\phi_{1}\right\|_{L^{\infty}[0, D]}<\frac{b}{2}, \\
\left\|c_{2}\right\|_{H^{2}[0, D]} \leq 2\left\|c_{1}\right\|_{H^{2}[0, D]} \\
\frac{1}{2} \frac{1}{c_{\max }} \leq \frac{1}{c_{2}(z)} \leq 2 \frac{1}{c_{\min }} \text { for all } 0 \leq z \leq D, \text { and } \\
\left|c_{2}(z)-c_{1}(z)\right| \leq c_{2}(z)+c_{1}(z) \text { for all } 0 \leq z \leq D .
\end{gathered}
$$

These assumptions assure us that the characteristics $t=\phi_{i}(z)$ corresponding to the two velocities are not too far apart and that the surface measurements depend on comparable depth intervals.

\subsection{Energy identities.}


2.2.1. The standard energy identity. Given a velocity $c(z)$ and smooth functions $u$ and $v$, we define the "standard" energy form $\eta$ to be

$$
\eta=\left[\frac{1}{c^{2}} \partial_{t} u \partial_{t} v+\partial_{z} u \partial_{z} v\right] d z+\left[\partial_{t} u \partial_{z} v+\partial_{z} u \partial_{t} v\right] d t
$$

Then

$$
d \eta=-\left(\partial_{t} u \square v+\partial_{t} v \square u\right) d z \wedge d t
$$

Now suppose $\tau, 0<z_{0}<z_{1}$, and $T=\tau+2 \phi\left(z_{1}\right)$ are given, and define $\Gamma$ to be

$$
\Gamma=\left\{(z, t) \mid z_{0} \leq z \leq z_{1}, \tau+\phi(z) \leq t \leq T-\phi(z)\right\}
$$

We define the operators $D^{+}$and $D^{-}$by $D^{ \pm}= \pm(1 / c) \partial_{i}+\partial_{z}$; these are the tangential derivatives along the characteristics. If we apply Stokes' theorem to $\eta$ over $\Gamma$, then we obtain

$$
\begin{gathered}
\int_{z_{0}}^{z_{1}}\left(D^{+} u D^{+} v\right)(z, \tau+\phi(z))-\int_{z_{0}}^{z_{1}}\left(D^{-} u D^{-} v\right)(z, T-\phi(z))= \\
\int_{\tau+\phi\left(z_{0}\right)}^{T-\phi\left(z_{0}\right)} d t\left[\partial_{t} u \partial_{z} v+\partial_{z} u \partial_{t} v\right]\left(z_{0}, t\right)-\int_{z_{0}}^{z_{1}} d z \int_{\tau+\phi(z)}^{T-\phi(z)} d t\left(\partial_{t} u \square v+\partial_{t} v \square u\right)
\end{gathered}
$$

In the case $u=v$ we obtain the standard identity:

$$
\begin{aligned}
\int_{z_{0}}^{z_{1}} & \left(D^{+} u(z, \tau+\phi(z))\right)^{2}-\int_{z_{0}}^{z_{1}}\left(D^{-} u(z, T-\phi(z))\right)^{2}= \\
& \int_{\tau+\phi\left(z_{0}\right)}^{T-\phi\left(z_{0}\right)} d t 2 \partial_{t} u\left(z_{0}, t\right) \partial_{z} u\left(z_{0}, t\right)-\int_{z_{0}}^{z_{1}} d z \int_{\tau+\phi(z)}^{T-\phi(z)} d t 2 \partial_{t} u \square u .
\end{aligned}
$$

2.2.2. The sideways energy identity. Next, for a smooth function $u$, we define the "sideways" energy $Q_{u}$ on $\Gamma$ to be

$$
Q_{u}(z)=\int_{\tau+\phi(z)}^{T-\phi(z)} d t\left[\left(\partial_{t} u(z, t)\right)^{2}+c^{2}(z)\left(\partial_{z} u(z, t)\right)^{2}\right]
$$

and the "sideways" energy form $\omega$ to be

$$
\omega=2\left[\partial_{t} u \partial_{z} u\right] d z+\left[\left(\partial_{t} u\right)^{2}+c^{2}\left(\partial_{z} u\right)^{2}\right] d t
$$

Then

$$
d \omega=\left[2 c c^{\prime}\left(\partial_{z} u\right)^{2}-2 c^{2}\left(\partial_{z} u \square u\right)\right] d z \wedge d t
$$

If we apply Stokes' theorem to $\omega$ over $\Gamma$, then we obtain the sideways energy identity:

$$
\begin{aligned}
Q_{u}\left(z_{0}\right)= & \int_{z_{0}}^{z_{1}} c(z)\left(D^{+} u(z, \tau+\phi(z))\right)^{2}+\int_{z_{0}}^{z_{1}} c(z)\left(D^{-} u(z, T-\phi(z))\right)^{2} \\
& +\int_{z_{0}}^{z_{1}} d z \int_{\tau+\phi(z)}^{T-\phi(z)} d t\left[2 c c^{\prime}\left(\partial_{z} u\right)^{2}-2 c^{2}\left(\partial_{z} u \square u\right)\right]
\end{aligned}
$$


2.3. The progressing wave expansion. In this section we will discuss the progressing wave expansion for solutions of the wave equation. Associated with the two velocities $c_{1}$ and $c_{2}$ are the two wave operators

$$
\square_{i}=\frac{1}{c_{i}^{2}(z)} \partial_{t t}^{2}-\partial_{z z}^{2}, \quad i=1,2 .
$$

We will first examine the solution $u_{i}$ of

$$
\begin{array}{rlrl}
\square_{i} u_{i} & =0, & z>0 \\
\partial_{z} u_{i}(0, t) & =-\delta(t) & \\
u_{i} & \equiv 0, \quad t<0 .
\end{array}
$$

Recall $\phi_{i}$, the travel-time to depth $z$ for the velocity $c_{i}(z)$ :

$$
\phi_{i}(z)=\int_{0}^{z} d \zeta \frac{1}{c_{i}(\zeta)}
$$

For smooth $c_{i}$, the progressing wave expansion [2] shows that $u_{i}$ will have a jump along the characteristic $t=\phi_{i}(z)$ of magnitude $a_{i}(z)=c_{i}^{\frac{1}{2}}(0) c_{i}^{\frac{1}{2}}(z)$. The progressing wave expansion expresses this in what amounts to a Taylor's series expansion valid on one side of the characteristic $t=\phi_{i}(z)$ :

$$
u_{i}(z, t)=\sum_{k=0}^{N} p_{k}(z) P_{k}\left(t-\phi_{i}(z)\right)+R_{N+1}(z, t) .
$$

The $P_{k}$ are given by

$$
P_{k}(x)=\left\{\begin{aligned}
0 & \text { if } x<0 \\
x^{k} & \text { if } x \geq 0
\end{aligned}\right.
$$

The coefficient $p_{0}(z)$ is the jump along the characteristic $t=\phi_{i}(z)$,

$$
p_{0}(z)=c_{i}^{\frac{1}{2}}(0) c_{i}^{\frac{1}{2}}(z)
$$

while the higher-order coefficients are found by solving the second transport equation,

$$
2 p_{k}^{\prime} \phi_{i}^{\prime}+p_{k} \phi_{i}^{\prime \prime}-p_{k-1}^{\prime \prime}=0
$$

We can easily solve (17). From the condition $\partial_{z} u_{i}(0, t)=-\delta(t)$ we find that

$$
p_{k}(0)=c_{i}(0) p_{k-1}^{\prime}(0)
$$

and so

$$
\begin{aligned}
p_{k}(z) & =c_{i}^{\frac{1}{2}}(z)\left[c_{i}^{\frac{1}{2}}(0) p_{k-1}^{\prime}(0)+\frac{1}{2} \int_{0}^{z} c_{i}^{\frac{1}{2}} p_{k-1}^{\prime \prime}\right] \\
& =\frac{1}{2} c_{i}^{\frac{1}{2}}(z)\left[\left(c_{i}^{\frac{1}{2}}(z) p_{k-1}^{\prime}(z)+c_{i}^{\frac{1}{2}}(0) p_{k-1}^{\prime}(0)\right)-\int_{0}^{z}\left(c_{i}^{\frac{1}{2}}\right)^{\prime} p_{k-1}^{\prime}\right]
\end{aligned}
$$


The remainder $R_{N+1}(z, t)$ is the solution of

$$
\begin{aligned}
\square_{i} R_{N+1} & =p_{N}^{\prime \prime}(z) P_{N}\left(t-\phi_{i}(z)\right), & & z>0 \\
\partial_{z} R_{N+1}(0, t) & =p_{N}^{\prime}(0) P_{N}(t) & & \\
R_{N+1} & \equiv 0, & & t<0 .
\end{aligned}
$$

For convenience, we will denote by $a_{i}$ and $b_{i}$ the first two coefficients in the progressing wave expansion of $u_{i}$ :

$$
\begin{aligned}
& a_{i}(z)=c_{i}^{\frac{1}{2}}(0) c_{i}^{\frac{1}{2}}(z) \\
& b_{i}(z)=\frac{1}{2} c_{i}^{\frac{1}{2}}(z)\left[\left(c_{i}^{\frac{1}{2}}(z) a_{i}^{\prime}(z)+c_{i}^{\frac{1}{2}}(0) a_{i}^{\prime}(0)\right)-\int_{0}^{z}\left(c_{i}^{\frac{1}{2}}\right)^{\prime} a_{i}^{\prime}\right] .
\end{aligned}
$$

One interpretation of the progressing wave expansion which will prove useful is that $u_{i}$ is the solution of the characteristic-initial-value problem

$$
\begin{array}{rlc}
\square_{i} u_{i} & =0, & t>\phi_{i}(z) \\
\partial_{z} u_{i}(0, t) & =0, & t>0 \\
u_{i}\left(z, \phi_{i}(z)\right) & =a_{i}(z) . &
\end{array}
$$

Higher derivatives of $u_{i}$ solve similar problems; we will also use the fact that $v_{i}=\partial_{t} u_{i}$ is the solution of the characteristic-initial-value problem

$$
\begin{aligned}
\square_{i} v_{i} & =0, & t>\phi_{i}(z) \\
\partial_{z} v_{i}(0, t) & =0, & t>0 \\
v_{i}\left(z, \phi_{i}(z)\right) & =b_{i}(z), &
\end{aligned}
$$

while $V_{i}=\partial_{t t}^{2} u_{i}$ solves

$$
\begin{aligned}
\square_{1} V_{i} & =0, & t>\phi_{i}(z) \\
\partial_{z} V_{i}(0, t) & =0, & t>0 \\
V_{i}\left(z, \phi_{i}(z)\right) & =h_{i}(z), &
\end{aligned}
$$

where the characteristic value $h_{i}(z)$ is the third coefficient in the expansion of $u_{i}$ :

$$
h_{i}(z)=\frac{1}{2} c_{i}^{\frac{1}{2}}(z)\left[\left(c_{i}^{\frac{1}{2}}(z) b_{i}^{\prime}(z)-c_{i}^{\frac{1}{2}}(0) b_{i}^{\prime}(0)\right)-\frac{1}{2} \int_{0}^{z}\left(c_{i}^{-\frac{1}{2}}\right)^{\prime} b_{i}^{\prime}\right] .
$$

A similar expansion exists for $\delta u_{i}$, the solution of the linearized problem (7) about $c=c_{i}$ :

$$
\delta u_{i}=-\delta \phi_{i}(z) a_{i}(z) \delta\left(t-\phi_{i}(z)\right)+\left(\delta a_{i}(z)-b_{i}(z) \delta \phi_{i}(z)\right) H\left(t-\phi_{i}(z)\right)+R(z, t),
$$

where $H$ is the Heaviside function, and

$$
\delta \phi_{i}(z)=-\int_{0}^{z} \frac{\delta c}{c_{i}^{2}}
$$

is the first-order change in the travel-time. The coefficient $\delta a_{i}$ is

$$
\delta a_{i}(z)=\frac{1}{2} \frac{\delta c(0)}{c_{i}^{\frac{1}{2}}(0)} c_{i}^{\frac{1}{2}}(z)+\frac{1}{2} c_{i}^{\frac{1}{2}}(0) \frac{\delta c(z)}{c_{i}^{\frac{1}{2}}(z)}
$$

and is the first-order change in $a_{i}$. For the remainder of the paper we will denote $\delta u_{1}$ simply by $\delta u$. 
2.4. The idea of the proof. Comparing terms from the progressing wave expansions for $u_{2}, u_{1}$, and $\delta u$, we see that

$$
\begin{aligned}
\left\|a_{2}-a_{1}\right\|_{L^{2}[0, D]} & \leq C\left\|c_{2}-c_{1}\right\|_{L^{2}[0, D]} \\
\left\|b_{2}-b_{1}\right\|_{L^{2}[0, D]} & \leq K\left\|c_{2}-c_{1}\right\|_{H^{2}[0, D]}
\end{aligned}
$$

In these estimates we are bounding the pointwise values of $c_{i}$ and its derivatives in terms of higher Sobolev norms. We also have

$$
\left\|a_{2}-a_{1}-\delta a\right\|_{L^{2}[0, D]} \leq C\left\|c_{2}-c_{1}\right\|_{H^{1}[0, D]}^{2} .
$$

Applying the standard interpolation result for Sobolev norms we obtain

$$
\left\|a_{2}-a_{1}-\delta a\right\|_{L^{2}[0, D]} \leq C\left\|c_{2}-c_{1}\right\|_{L^{2}[0, D]}\left\|c_{2}-c_{1}\right\|_{H^{2}[0, D]} .
$$

The constant $C$ depends on $\left\|c_{1}\right\|_{H^{1}[0, D]}$,

$$
m_{1}=\min _{0 \leq z \leq D} c_{1}(z)
$$

$D$, and $r$, while the constant $K$ depends on $\left\|c_{1}\right\|_{H^{2}[0, D]}, m_{1}, D$, and $r$.

On the one hand, these estimates show that the velocity perturbation $\delta c$ causes only a small difference, commensurate with the size of $\delta c$, in the values of the $u_{i}$ along the characteristics $t=\phi_{i}(z)$. Unfortunately, the characteristics associated with the velocities $c_{i}$ have changed as well, which, as described in the introduction, results in a severe nonlinearity. In particular, $u_{2}-u_{1}$ and $u_{2}-u_{1}-\delta u$ do not solve characteristic-initialvalue problems but instead only initial-boundary-value problems with distribution data. This makes a direct estimate of $u_{2}-u_{1}$ and $u_{2}-u_{1}-\delta u$ inaccessible.

We will instead prove the estimates in Theorems 1.1 and 1.2 indirectly. If we can show that

$$
\left|\left\langle f, F\left(c_{2}\right)-F\left(c_{1}\right)\right\rangle_{L^{2}[0, T]}\right| \leq K\left\|c_{2}-c_{1}\right\|_{H^{1}[0, D]}\|f\|_{L^{2}[0, T]}
$$

for all $f \in C_{0}^{\infty}(0, T)$, then we can conclude that

$$
\left\|F\left(c_{2}\right)-F\left(c_{1}\right)\right\|_{L^{2}[0, T]} \leq K\left\|c_{2}-c_{1}\right\|_{H^{1}[0, D]},
$$

whirh in the Lipschitz estimate of Theorem 1.1.

Similarly, an estimate of the form

$$
\begin{aligned}
& \left|\left\langle f, F\left(c_{2}\right)-F\left(c_{1}\right)-D F\left[c_{1}\right](\delta c)\right\rangle_{L^{2}[0, T]}\right| \\
& \quad \leq\left[K\|\delta c\|_{L^{2}[0, D]}\|\delta c\|_{H^{2}[0, D]}+B\|\delta c\|_{L^{2}}^{2}\right]\|f\|_{L^{2}}
\end{aligned}
$$

for all $f \in C_{0}^{\infty}(0, T)$ yields the estimate in Theorem 1.2.

The idea of the proof, then, is to derive bounds of the form (28) and (29). To this end, suppose that $w$ solves

$$
\begin{array}{rlrl}
\square_{1} w & =0, & z>0 \\
\partial_{z} w(0, t) & =f(t), \quad f \in C_{0}^{\infty}(0, T) \\
w & \equiv 0, \quad t \geq T-\phi_{1}(z) .
\end{array}
$$


If we apply the Stokes' theorem identity (15) with $u=u_{i}$ and $v=w$ over the region $\left\{(z, t) \mid 0 \leq z \leq D, \phi_{i}(z) \leq t \leq 2 \phi_{i}(D)-\phi_{i}(z)\right\}$, we obtain the following formulae for $\left\langle f, F\left(c_{i}\right)\right\rangle_{L^{2}[0, T]}$.

$$
\begin{gathered}
\int_{0}^{T} d t f(t) \partial_{t} u_{1}(0, t)=\int_{0}^{D} d z D_{1}^{+} w\left(z, \phi_{1}(z)\right) \frac{d}{d z}\left(u_{1}\left(z, \phi_{1}(z)\right)\right) \\
\int_{0}^{T} d t f(t) \partial_{t} u_{2}(0, t)=\int_{0}^{D} d z D_{2}^{+} w\left(z, \phi_{2}(z)\right) \frac{d}{d z}\left(u_{2}\left(z, \phi_{2}(z)\right)\right) \\
-\int_{0}^{D} d z \int_{\phi_{2}(z)}^{T-\phi_{1}(z)} d t \partial_{t} u_{2}(z, t) \square_{2} w(z, t)
\end{gathered}
$$

From these identities we see that we will need to estimate the difference of the traces of solutions of the wave equation restricted to the two nearby characteristics $t=\phi_{i}(z)$, $i=1,2$. This is the subject of the next sections.

2.5. Trace estimates. In this section we present estimates for the traces of solutions of the wave equation along characteristics. The results of this section are propagation of regularity results for the wave equation, with explicit dependence on the smoothness of the velocity coefficient. These trace estimates show that the solutions of the wave equation are a great deal smoother than the usual trace theorem would lead us to believe.

These trace estimates hinge on two facts. The first is that the wave operator in one spatial dimension $z$ is hyperbolic in both $t$ and $z$, a consequence of which is the sideways energy identity of Section 2.2.

The second point is that we can almost factor the wave operator into derivatives along its characteristics. For $i=1,2$, let

$$
\begin{aligned}
& D_{i}^{+}=\frac{1}{c_{i}} \partial_{t}+\partial_{z} \\
& D_{i}^{-}=-\frac{1}{c_{i}} \partial_{t}+\partial_{z}
\end{aligned}
$$

These are the tangential derivatives along the curves $t=\tau \pm \phi_{i}(z)$. Then

$$
\begin{aligned}
& D_{i}^{+} D_{i}^{-}=-\square_{i}+\frac{c_{i}^{\prime}}{c_{i}^{2}} \partial_{t} \\
& D_{i}^{-} D_{i}^{+}=-\square_{i}-\frac{c_{i}^{\prime}}{c_{i}^{2}} \partial_{t} .
\end{aligned}
$$

One consequence of these identities is the commutator relation

$$
D_{i}^{+} D_{i}^{-}=D_{i}^{-} D_{i}^{+}+\frac{2 c_{i}^{\prime}(z)}{c_{i}^{2}(z)} \partial_{t}
$$

Equations (32) and (33) indicate that we can factor the wave operator, up to a lower-order term, into tangential derivatives along the forward and backward characteristics. This fact will enable us to derive sharp estimates in these directions for traces of solutions of the wave equation. 
We will use the following conventions for the remainder of this paper. Recall the depth $D$ and $r$ from Section 2.1. Given a velocity $c_{i}, i=1,2$, let

$$
\begin{aligned}
m_{i} & =\min _{0 \leq z \leq D} c_{i}(z) \\
M_{i} & =\max _{0 \leq z \leq D} c_{i}(z) .
\end{aligned}
$$

$C_{i}, K_{i}$, and $B_{i}$ will represent various constants whose value in the proofs that follow will change, even from line to line, but will be consistent insofar as

$$
\begin{aligned}
C_{i} & =C_{i}\left(\left\|c_{i}\right\|_{H^{1}[0, D]}, m_{i}, M_{i}, D, r\right), \\
K_{i} & =K_{i}\left(\left\|c_{i}\right\|_{H^{2}[0, D]}, m_{i}, M_{i}, D, r\right), \text { and } \\
B_{i} & =B_{i}\left(\left\|c_{i}\right\|_{H^{3}[0, D]}, m_{i}, M_{i}, D, r\right) .
\end{aligned}
$$

The dependence on the maximum $M_{i}$ is mentioned only for clarity since we can replace $M_{i}$ in this one-dimensional problem with $\left\|c_{i}\right\|_{H^{1}[0, D]}$.

We will also often suppress the domains over which norms are computed. Unless otherwise noted, the norm of functions of $z$ alone are taken over the interval $[0, D]$, while those of functions of $t$ only are taken over the interval $[0, T]$.

Finally, recall $b$ from Section 2.1. Given $\tau \geq-b / 2$, set $T_{i}=\tau+2 \phi_{i}(D)$ and define the domain $\Gamma$ to be

$$
\Gamma \equiv \Gamma_{\tau}=\left\{(z, t) \mid 0 \leq z \leq D, \tau+\phi_{i}(z) \leq t \leq T_{i}-\phi_{i}(z)\right\}
$$

Associated with $\Gamma$ is the sideways energy $Q_{u, \tau}$ :

$$
Q_{u, \tau}(z)=\int_{\tau+\phi_{i}(z)}^{T_{i}-\phi_{i}(z)} d t\left[\left(\partial_{t} u(z, t)\right)^{2}+c_{i}^{2}(z)\left(\partial_{z} u(z, t)\right)^{2}\right]
$$

We begin with a general proposition relating the sideways energy $Q_{u, \tau}$ of a function $u$, the tangential derivatives $D_{i}^{+} u$ and $D_{i}^{-} u$, and $\square_{i} u$.

Proposition 2.1. Suppose that $u$ is smooth on $\Gamma$. Then there exists a constant $C_{i}$ such that

$$
\begin{aligned}
& \left\|D_{i}^{+} u\left(z, \tau+\phi_{i}(z)\right)\right\|_{L^{2}[0, D]} \leq \\
& C_{i}\left[\left\|D_{i}^{-} u\left(z, T_{i}-\phi_{i}(z)\right)\right\|_{L^{2}[0, D]}+\left\|\partial_{z} u(0, t)\right\|_{L^{2}\left[0, T_{i}\right]}+\left\|\square_{i} u\right\|_{L^{2}(\Gamma)}\right], \\
& \left\|D_{i}^{-} u\left(z, T_{i}-\phi_{i}(z)\right)\right\|_{L^{2}[0, D]} \leq \\
& \quad C_{i}\left[\left\|D_{i}^{+} u\left(z, \tau+\phi_{i}(z)\right)\right\|_{L^{2}[0, D]}+\left\|\partial_{z} u(0, t)\right\|_{L^{2}\left[0, T_{i}\right]}+\left\|\square_{i} u\right\|_{\left.L^{2}(\Gamma)\right], \text { and }}\right] \\
& \left\|Q_{u, \tau}^{\frac{1}{2}}\right\|_{L^{\infty}[0, D]} \leq \\
& C_{i}\left[\left\|D_{i}^{+} u\left(z, \tau+\phi_{i}(z)\right)\right\|_{L^{2}[0, D]}+\left\|\partial_{z} u(0, t)\right\|_{L^{2}\left[0, T_{i}\right]}+\left\|\square_{i} u\right\|_{L^{2}(\Gamma)}\right] .
\end{aligned}
$$


Proof. Let $z \in[0, D]$. If we integrate the sideways energy form of Section 2.2 .2 around the boundary of $\left\{(\zeta, t) \mid z \leq \zeta \leq D, \tau+\phi_{i}(\zeta) \leq t \leq T_{i}-\phi_{i}(\zeta)\right\}$, we obtain

$$
\begin{gathered}
Q_{u, \tau}(z)=\int_{z}^{D} d \zeta c_{i}(\zeta)\left(D_{i}^{+} u\left(\zeta, \tau+\phi_{i}(\zeta)\right)\right)^{2}+\int_{z}^{D} d \zeta c_{i}(\zeta)\left(D^{-} u\left(\zeta, T_{i}-\phi_{i}(\zeta)\right)\right)^{2} \\
\quad+\int_{z}^{D} d \zeta \int_{\tau+\phi_{i}(\zeta)}^{T_{i}-\phi_{i}(\zeta)} d t\left[2 c_{i}(\zeta) c_{i}^{\prime}(\zeta)\left(\partial_{\zeta} u(\zeta, t)\right)^{2}-2 c_{i}^{2}(\zeta)\left(\partial_{\zeta} u(\zeta, t) \square_{i} u(\zeta, t)\right)\right]
\end{gathered}
$$

and thus

$$
\begin{gathered}
Q_{u, \tau}(z) \leq\|c\|_{L^{\infty}}\left[\left\|D_{i}^{+} u\left(z, \tau+\phi_{i}(z)\right)\right\|_{L^{2}}^{2}+\left\|D^{-} u\left(z, T_{i}-\phi_{i}(z)\right)\right\|_{L^{2}}^{2}\right] \\
+\int_{z}^{D} d \zeta\left(1+2\left|\frac{c_{i}^{\prime}(\zeta)}{c_{i}(\zeta)}\right|\right) Q_{u, \tau}(\zeta)+\left\|c_{i}^{2}\right\|_{L^{\infty}}\left\|\square_{i} u\right\|_{L^{2}}^{2} .
\end{gathered}
$$

Applying Gronwall's inequality, we arrive at the sideways estimate

$$
Q_{u, \tau}(z) \leq \lambda\left[\left\|D_{i}^{+} u\left(z, \tau+\phi_{i}(z)\right)\right\|_{L^{2}}^{2}+\left\|D^{-} u\left(z, T_{i}-\phi_{i}(z)\right)\right\|_{L^{2}}^{2}+\left\|\square_{i} u\right\|_{L^{2}}^{2}\right]
$$

where

$$
\lambda=\left(\left\|c_{i}\right\|_{L^{\infty}}+\left\|c_{i}^{2}\right\|_{L^{\infty}}\right) \exp \left(\left\|1+2\left|\frac{c_{i}^{\prime}(\zeta)}{c_{i}(\zeta)}\right|\right\|_{L^{1}}\right)
$$

Meanwhile, the standard identity (15) is

$$
\begin{aligned}
\int_{0}^{D} d z\left(D_{i}^{+} u\left(z, \tau+\phi_{i}(z)\right)\right)^{2} & =\int_{0}^{D} d z\left(D_{i}^{-} u\left(z, T_{i}-\phi_{i}(z)\right)\right)^{2} \\
\int_{0}^{T} d t \partial_{z} u(0, t) \partial_{t} u(0, t) & +\int_{0}^{D} d z \int_{\tau+\phi_{i}(z)}^{T_{i}-\phi_{i}(z)} d t 2 \partial_{t} u(z, t) \square_{i} u(z, t)
\end{aligned}
$$

whence

$$
\begin{aligned}
& \int_{0}^{D} d z\left(D_{i}^{+} u(z, \phi(z))\right)^{2} \leq \\
& \quad\left\|D_{i}^{-} u\left(z, T_{i}-\phi_{i}(z)\right)\right\|_{L^{2}}^{2}+\int_{0}^{T} d t \frac{1}{\alpha^{2}}\left(\partial_{z} u(0, t)\right)^{2}+\alpha^{2}\left(\partial_{t} u(0, t)\right)^{2} \\
& \quad+\int_{0}^{D} d z \int_{\phi(z)}^{T-\phi(z)} d t\left[\frac{1}{\alpha^{2}}\left(\square_{i} u(z, t)\right)^{2}+\alpha^{2}\left(\partial_{t} u(z, t)\right)^{2}\right]
\end{aligned}
$$

where $\alpha$ is a real number whose value will be chosen shortly. From this inequality we immediately obtain

$$
\begin{aligned}
\int_{0}^{D} d z\left(D_{i}^{+} u\left(z, \tau+\phi_{i}(z)\right)\right)^{2} & \leq\left\|D_{i}^{-} u\left(z, T_{i}-\phi_{i}(z)\right)\right\|_{L^{2}}^{2} \\
& \frac{1}{\alpha^{2}}\left(\left\|\partial_{z} u(0, t)\right\|_{L^{2}}^{2}+\left\|\square_{i} u\right\|_{L^{2}}^{2}\right)+\alpha^{2}\left(Q_{u, \tau}(0)+\int_{0}^{D} d z Q_{u, \tau}(z)\right)
\end{aligned}
$$


Now we use the bound on $Q_{u, \tau}(z)$ from (38):

$$
\begin{aligned}
& \int_{0}^{D} d z\left(D_{i}^{+} u\left(z, \tau+\phi_{i}(z)\right)\right)^{2} \leq \\
& \left\|D_{i}^{-} u\left(z, T_{i}-\phi_{i}(z)\right)\right\|_{L^{2}}^{2}+\frac{1}{\alpha^{2}}\left(\left\|\partial_{z} u(0, t)\right\|_{L^{2}}^{2}+\left\|\square_{i} u\right\|_{L^{2}}^{2}\right) \\
& +\alpha^{2}(1+D) \lambda\left[\left\|D_{i}^{+} u\left(z, \tau+\phi_{i}(z)\right)\right\|_{L^{2}}^{2}+\left\|D_{i}^{-} u\left(z, T_{i}-\phi_{i}(z)\right)\right\|_{L^{2}}^{2}+\left\|\square_{i} u\right\|_{L^{2}}^{2}\right] .
\end{aligned}
$$

Set $\alpha^{2}=\frac{1}{2}((1+D) \lambda)^{-1}$. Then $(40)$ yields

$$
\begin{gathered}
\frac{1}{2} \int_{0}^{D} d z\left(D_{i}^{+} u\left(z, \tau+\phi_{i}(z)\right)\right)^{2} \leq \frac{3}{2}\left\|D_{i}^{-} u\left(z, T_{i}-\phi_{i}(z)\right)\right\|_{L^{2}}^{2} \\
+\frac{1}{\alpha^{2}}\left(\left\|\partial_{z} u(0, t)\right\|_{L^{2}}^{2}+\left\|\square_{i} u\right\|_{L^{2}}^{2}\right)+\frac{1}{2}\left\|\square_{i} u\right\|_{L^{2}}^{2},
\end{gathered}
$$

from which (35) follows.

In exactly the same way we can start with (39) and arrive at (36):

$$
\begin{aligned}
& \int_{0}^{D} d z\left(D_{i}^{-} u\left(z, T_{i}-\phi_{i}(z)\right)\right)^{2} \leq \\
& \quad C_{i}\left[\left\|D_{i}^{+} u\left(z, \tau+\phi_{i}(z)\right)\right\|_{L^{2}}^{2}+\left\|\partial_{z} u(0, t)\right\|_{L^{2}}^{2}+\left\|\square_{i} u\right\|_{L^{2}}\right] .
\end{aligned}
$$

Substituting this last estimate into (38) we obtain

$$
Q_{u, \tau}(z) \leq C_{i}\left[\left\|D_{i}^{+} u\left(z, \tau+\phi_{i}(z)\right)\right\|_{L^{2}}^{2}+\left\|\partial_{z} u(0, t)\right\|_{L^{2}}^{2}+\left\|\square_{i} u\right\|_{L^{2}}^{2}\right]
$$

which is $(37)$.

Recall $u_{i}$, the solution of $(6)$ with $c=c_{i}$. We will use the preceding proposition with $\tau=0$ to bound the energy of $u_{i}, \partial_{t} u_{i}$, and $\partial_{t t}^{2} u_{i}$ on vertical strips.

Proposition 2.2. There exist constants $C_{i}, K_{i}$, and $B_{i}$ such that for all $z \in[0, D]$,

$$
\begin{gathered}
\int_{\phi_{i}(z)}^{2 \phi_{i}(D)-\phi_{i}(z)} d t\left(\partial_{t} u_{i}(z, t)\right)^{2} \leq C_{i} \\
\int_{\phi_{i}(z)}^{2 \phi_{i}(D)-\phi_{i}(z)} d t\left(\partial_{t t}^{2} u_{i}(z, t)\right)^{2} \leq K_{i} \\
\int_{\phi_{i}(z)}^{2 \phi_{i}(D)-\phi_{i}(z)} d t\left(\partial_{t t t}^{3} u_{i}(z, t)\right)^{2} \leq B_{i}
\end{gathered}
$$

Proof. In Section 2.3 we saw that $u_{i}, \partial_{t} u_{i}$, and $\partial_{t t}^{2} u_{i}$ solve the characteristic-initialvalue problems $(21)-(23)$, where the values along the characteristic $t=\phi_{i}(z)$ are explicitly known. This together with (37) yield (41) - (43).

The estimate (41) shows that the map $F: c \mapsto \partial_{t} u(0, t)$ extends as a map $H^{1} \rightarrow L^{2}$. Thus the boundary value $\partial_{t} u(0, t)$ in problem (6) with distribution datum is defined for velocities in $H^{1}$. 
The estimates of the Proposition 2.1 can be both simplified and strengthened for a class of solutions significant to our proof.

Proposition 2.3. Suppose that $u$ is smooth and that $u$ vanishes for $t \geq T-\phi_{1}(z)$. Then there exists a constant $C_{i}$ such that

$$
\begin{gathered}
\left\|D_{i}^{+} u\left(z, \tau+\phi_{i}(z)\right)\right\|_{L^{2}[0, D]} \leq C_{i}\left[\left\|\partial_{z} u(0, t)\right\|_{L^{2}\left[0, T_{i}\right]}+\left\|\square_{i} u\right\|_{L^{2}(\Gamma)}\right] \\
\left\|Q_{u, \tau}^{\frac{1}{2}}\right\| \|_{L^{\infty}[0, D]} \leq C_{i}\left[\left\|\partial_{z} u(0, t)\right\|_{L^{2}\left[0, T_{i}\right]}+\left\|\square_{i} u\right\|_{L^{2}(\Gamma)}\right] \\
\left\|D_{i}^{-} u\right\|_{L^{\infty}(\Gamma)} \leq C_{i}\left[\left\|\partial_{z} u(0, t)\right\|_{L^{2}\left[0, T_{i}\right]}+\sup _{\sigma \geq \tau}\left\|\square_{i} u\left(z, \sigma+\phi_{i}(z)\right)\right\|_{L^{2}[0, D]}\right] .
\end{gathered}
$$

Proof. In Section 2.1 we arranged matters so that

$$
T_{i}-\phi_{i}(z) \geq T-\phi_{1}(z)
$$

for all $0 \leq z \leq D$, so $D_{i}^{-} u\left(z, T_{i}-\phi_{i}(z)\right) \equiv 0$. The estimates (44) and (45) then follow from (35) and (36).

In order to bound the $L^{\infty}$-norm of $D_{i}^{-} u$, we begin with (32). For $(z, t) \in \Gamma$, let $t=\sigma+\phi_{i}(z)$. From (32) we have

$$
D_{i}^{+} D_{i}^{-} u=\frac{c_{i}^{\prime}}{c_{i}^{2}} \partial_{t} u-\square_{i} u=\frac{c_{i}^{\prime}}{c_{i}^{2}} \frac{c_{i}}{2}\left(D_{i}^{+} u-D_{i}^{-} u\right)-\square_{i} u
$$

which we write out as an ordinary differential equation along a characteristic:

$$
\begin{gathered}
\frac{d}{d z}\left[D_{i}^{-} u\left(z, \sigma+\phi_{i}(z)\right)\right]+\frac{1}{2} \frac{c_{i}^{\prime}}{c_{i}} D_{i}^{-} u\left(z, \sigma+\phi_{i}(z)\right)= \\
\frac{1}{2} \frac{c_{i}^{\prime}}{c_{i}} D_{i}^{+} u\left(z, \sigma+\phi_{i}(z)\right)-\square_{i} u\left(z, \sigma+\phi_{i}(z)\right) .
\end{gathered}
$$

An integrating factor for this differential equation in $D_{i}^{-} u$ is $c_{i}^{\frac{1}{2}}$ :

$$
\frac{d}{d z}\left[c_{i}^{\frac{1}{2}}(z) D_{i}^{-} u\left(z, \sigma+\phi_{i}(z)\right)\right]=\left(c_{i}^{\frac{1}{2}}\right)^{\prime}(z) D_{i}^{+} u\left(z, \sigma+\phi_{i}(z)\right)-c_{i}^{\frac{1}{2}}(z) \square_{i} u\left(z, \sigma+\phi_{i}(z)\right) .
$$

Since the depth $D$ is deep enough that $D_{i}^{-} u(D, \sigma+\phi(D))=0$, upon integration we obtain

$$
c^{\frac{1}{2}}(z) D_{i}^{-} u(z, t)=\int_{D}^{z} d \zeta\left(c^{\frac{1}{2}}\right)^{\prime}(\zeta) D_{i}^{+} w\left(\zeta, \sigma+\phi_{i}(\zeta)\right)-c^{\frac{1}{2}}(\zeta) \square_{i} u\left(\zeta, \sigma+\phi_{i}(\zeta)\right)
$$

and thus

$$
\left\|D_{i}^{-} u\right\|_{L^{\infty}(\Gamma)} \leq C_{i} \sup _{\sigma \geq \tau}\left[\left\|D_{i}^{+} u\left(z, \sigma+\phi_{i}(z)\right)\right\|_{L^{2}[0, D]}+\left\|\square_{i} u\left(z, \sigma+\phi_{i}(z)\right)\right\|_{L^{2}[0, D]}\right] .
$$


Then (46) follows from (44), since the latter assures us that

$$
\sup _{\sigma \geq \tau}\left\|D_{i}^{+} u\left(z, \sigma+\phi_{i}(z)\right)\right\|_{L^{2}[0, D]} \leq C_{i}\left[\left\|\partial_{z} u(0, t)\right\|_{L^{2}\left[0, T_{i}\right]}+\left\|\square_{i} u\right\|_{L^{2}(\Gamma)}\right] .
$$

We will now begin to apply the preceding estimates. Recall $w$, which is the solution of

$$
\begin{aligned}
\square_{1} w & =0 \\
\partial_{z} w(0, t) & =f(t), \quad f \in C_{0}^{\infty}(0, T) \\
w & \equiv 0, \quad t \geq T-\phi_{1}(z)
\end{aligned}
$$

Proposition 2.4. There exist constants $C_{1}$ and $K_{1}$ such that

$$
\begin{aligned}
\left\|D_{1}^{+} w\left(z, \tau+\phi_{1}(z)\right)\right\|_{L^{2}[0, D]} & \leq C_{1}\|f\|_{L^{2}[0, T]} \\
\left\|Q_{w, \tau}^{\frac{1}{2}}\right\|_{L^{\infty}[0, D]} & \leq C_{1}\|f\|_{L^{2}[0, T]} \\
\left\|D_{1}^{-} w\right\|_{L^{\infty}(\Gamma)} & \leq C_{1}\|f\|_{L^{2}[0, T]} \\
\left\|\partial_{t} w\left(z, \tau+\phi_{1}(z)\right)\right\|_{L^{2}[0, D]} & \leq C_{1}\|f\|_{L^{2}[0, T]} \\
\left\|D_{1}^{-} D_{1}^{-} w\right\|_{L^{\infty}(\Gamma)} & \leq K_{1}\|f\|_{L^{2}[0, T]}
\end{aligned}
$$

Proof. The bounds (48) - (50) follow from Proposition 2.3. The estimate (51) follows from (48), (50), and the relation $\partial_{t}=\frac{1}{2} c_{1}\left(D_{1}^{+}-D_{1}^{-}\right)$.

To derive (52), we begin with the following relation, which is a consequence of the commutator relation (34) and $\partial_{t}=\frac{1}{2} c_{1}\left(D_{1}^{+}-D_{1}^{-}\right)$:

$$
D_{1}^{+} D_{1}^{-} D_{1}^{-} w+\frac{3 c_{1}^{\prime}(z)}{c_{1}^{2}(z)} \frac{c_{1}}{2} D_{1}^{-} D_{1}^{-} w=\left[\frac{3}{2}\left(\frac{c_{1}^{\prime}(z)}{c_{1}^{2}(z)}\right)^{2}-\left(\frac{1}{c_{1}}\right)^{\prime \prime}(z)\right] \partial_{t} w
$$

An integrating factor for this equation is $c_{1}^{\frac{3}{2}}(z)$ :

$$
\begin{aligned}
& \frac{d}{d z}\left[c_{1}^{\frac{3}{2}}(z) D_{1}^{-} D_{1}^{-} w\left(z, \tau+\phi_{1}(z)\right)\right] \\
& \quad=c_{1}^{\frac{3}{2}}(z)\left[\frac{3}{2}\left(\frac{c_{1}^{\prime}(z)}{c_{1}^{2}(z)}\right)^{2}-\left(\frac{1}{c_{1}}\right)^{\prime \prime}(z)\right] \partial_{t} w\left(z, \tau+\phi_{1}(z)\right)
\end{aligned}
$$

Integration then yields

$$
\begin{aligned}
& D_{1}^{-} D_{1}^{-} w(z, t)= \\
& \quad c_{1}^{-\frac{3}{2}}(z) \int_{D}^{z} d \zeta c_{1}^{\frac{3}{2}}(\zeta)\left[\frac{3}{2}\left(\frac{c_{1}^{\prime}(\zeta)}{c_{1}^{2}(\zeta)}\right)^{2}-\left(\frac{1}{c_{1}}\right)^{\prime \prime}(\zeta)\right] \partial_{t} w\left(\zeta, \tau+\phi_{i}(\zeta)\right)
\end{aligned}
$$


Then from (51),

$$
\left\|D_{1}^{-} D_{1}^{-} w\right\|_{L^{\infty}} \leq K_{1}\|f\|_{L^{2}}
$$

which is (52).

We can also estimate traces along curves which are not characteristics; in particular, we can obtain trace estimates for solutions associated with $c_{1}$ along the characteristics associated with $c_{2}$, and vice versa.

Proposition 2.5. There exists a constant $C_{1}$ such that

$$
\begin{aligned}
\left\|D_{2}^{+} w\left(z, \phi_{2}(z)\right)\right\|_{L^{2}[0, D]} & \leq C_{1}\|f\|_{L^{2}[0, T]} \\
\left\|\partial_{t} w\left(z, \phi_{2}(z)\right)\right\|_{L^{2}[0, D]} & \leq C_{1}\|f\|_{L^{2}[0, T]}
\end{aligned}
$$

Proof. We will prove (53) first. Apply Stokes' theorem to the standard energy form of Section 2.2.1 over the region $\left\{(z, t) \mid 0 \leq z \leq D, \phi_{2}(z) \leq t \leq \phi_{1}(D)+\phi_{2}(D)-\phi_{1}(z)\right\}$. Since $\phi_{1}(D)+\phi_{2}(D)-\phi_{1}(z) \geq T-\phi_{1}(z)$ for $0 \leq z \leq D$,

$$
D_{1}^{--} w\left(z, \phi_{1}(D)+\phi_{2}(D)-\phi_{1}(z)\right) \equiv 0
$$

and we obtain

$$
\begin{aligned}
& \int_{0}^{\phi_{1}(D)+\phi_{2}(D)} d t \partial_{z} w(0, t) \partial_{t} w(0, t) \\
& \quad=\int_{0}^{D} d z\left(D_{2}^{+} w\left(z, \phi_{2}(z)\right)\right)^{2}+\left(\left(\phi_{1}^{\prime}(z)\right)^{2}-\left(\phi_{2}^{\prime}(z)\right)^{2}\right)\left(\partial_{t} w\left(z, \phi_{2}(z)\right)\right)^{2} .
\end{aligned}
$$

Since $D_{2}^{+}-D_{1}^{-}=\left(\phi_{2}^{\prime}+\phi_{1}^{\prime}\right) \partial_{t}$, the preceding yields

$$
\begin{aligned}
& \int_{0}^{\phi_{1}(D)+\phi_{2}(D)} d t \partial_{z} w(0, t) \partial_{t} w(0, t) \\
& \quad=\int_{0}^{D} d z\left(D_{2}^{+} w\left(z, \phi_{2}(z)\right)\right)^{2}+\frac{\phi_{1}^{\prime}-\phi_{2}^{\prime}}{\phi_{1}^{\prime}+\phi_{2}^{\prime}}\left(D_{2}^{+} w\left(z, \phi_{2}(z)\right)-D_{1}^{-} w\left(z, \phi_{2}(z)\right)\right)^{2} \\
& \quad \geq \int_{0}^{D} d z\left(D_{2}^{+} w\left(z, \phi_{2}(z)\right)\right)^{2}-2 \frac{\phi_{1}^{\prime}-\phi_{2}^{\prime}}{\phi_{1}^{\prime}+\phi_{2}^{\prime}}\left(\left(D_{2}^{+} w\left(z, \phi_{2}(z)\right)\right)^{2}+\left(D_{1}^{-} w\left(z, \phi_{2}(z)\right)\right)^{2}\right)
\end{aligned}
$$

By hypothesis (13),

$$
\left|\frac{\phi_{1}^{\prime}-\phi_{2}^{\prime}}{\phi_{1}^{\prime}+\phi_{2}^{\prime}}\right| \leq \frac{1}{4}
$$

so

$\frac{1}{2} \int_{0}^{D} d z\left(D_{2}^{+} w\left(z, \phi_{2}(z)\right)\right)^{2} \leq \int_{0}^{D} d z\left(D_{1}^{-} w\left(z, \phi_{2}(z)\right)\right)^{2}+\int_{0}^{\phi_{1}(D)+\phi_{2}(D)} d t \partial_{z} w(0, t) \partial_{t} w(0, t)$.

The estimate (53) then follows from the estimates in Proposition 2.4. To obtain (54), note that $D_{2}^{+}-D_{1}^{-}=\left(\phi_{2}^{\prime}+\phi_{1}^{\prime}\right) \partial_{t}$ and use (43) and (53). 
Using Propositions 2.4 and 2.5, we can estimate the traces of solutions in which $w$ appears as a datum.

Proposition 2.6. Suppose that $v$ solves

$$
\begin{aligned}
\square_{2} v & =a(z) \partial_{t} w \\
\partial_{z} v(0, t) & =0 \\
v & \equiv 0, \quad t \geq T-\phi_{1}(z)
\end{aligned}
$$

where $a(z)$ is a smooth function.

Then there exist constants $C_{2}$ and $K_{2}$ such that

$$
\begin{aligned}
\left\|D_{2}^{+} v\left(z, \tau+\phi_{i}(z)\right)\right\|_{L^{2}[0, D]} & \leq C_{2}\|a\|_{L^{2}[0, D]}\|f\|_{L^{2}[0, T]} \\
\left\|Q_{v, \tau}^{\frac{1}{2}}\right\|_{L^{\infty}[0, D]} & \leq C_{2}\|a\|_{L^{2}[0, D]}\|f\|_{L^{2}[0, T]} \\
\left\|D_{2}^{-} v\right\|_{L^{\infty}(\Gamma)} & \leq K_{2}\|a\|_{L^{2}[0, D]}\|f\|_{L^{2}[0, T]} \\
\left\|\partial_{t} v\left(z, \phi_{1}(z)\right)\right\|_{L^{2}[0, D]} & \leq C_{2}\|a\|_{L^{2}[0, D]}\|f\|_{L^{2}[0, T]}
\end{aligned}
$$

Proof. The bounds (56) - (58) follow from the estimates in Proposition 2.3, 2.4, and 2.5. The key is that the bounds in Propositions 2.4 and 2.5 for $w$ enable us to bound the $L^{2}$-norm of $\partial_{t} w$ and thereby $\square_{i} v$ both on $\Gamma$ and along characteristics. The proof of the noncharacteristic trace estimate (59) follows the same line of argument as the proof of Proposition 2.5.

Finally, there is one other function whose traces we will need to examine. This function arises when integrating the function $w$ along characteristics. For $(z, t), 0 \leq$ $z \leq D$, define

$$
W(z, t)=\int_{D}^{z} d \zeta w\left(\zeta, t-\phi_{1}(z)+\phi_{1}(\zeta)\right)
$$

This is the integral of $w$ along the characteristic through $(z, t)$. As we would expect, it is one degree smoother than $w$ in one characteristic direction and as smooth as $w$ in the other.

Proposition 2.7. There exist constants $C_{1}$ and $K_{1}$ such that

$$
\begin{aligned}
\left\|D_{1}^{+} D_{1}^{+} W\left(z, \tau+\phi_{1}(z)\right)\right\|_{L^{2}[0, D]} & \leq C_{1}\|f\|_{L^{2}[0, T]} \\
\left\|D_{1}^{-} D_{1}^{-} W\right\|_{L^{\infty}(\Gamma)} & \leq K_{1}\|f\|_{L^{2}[0, T]} \\
\left\|D_{1}^{+} \partial_{t} W\left(z, \tau+\phi_{1}(z)\right)\right\|_{L^{2}[0, D]} & \leq C_{1}\|f\|_{L^{2}[0, T]} \\
\left\|D_{1}^{-} \partial_{t} W\left(z, \tau+\phi_{1}(z)\right)\right\|_{L^{\infty}[0, D]} & \leq K_{1}\|f\|_{L^{2}[0, T]} .
\end{aligned}
$$

Proof. The bounds (61) and (63) follow from

$$
\begin{aligned}
D_{1}^{+} D_{1}^{+} W(z, t) & =D_{1}^{+} w(z, t) \\
D_{1}^{+} \partial_{t} W(z, t) & =\partial_{t} w(z, t)
\end{aligned}
$$


and the estimates in Proposition 2.4.

$L^{\infty}$-bounds on $D_{1}^{-} W$ and $D_{1}^{-} D_{1}^{-} W$ follow from the fact that if

$$
U(z, t)=\int_{D}^{z} d \zeta u\left(\zeta, t-\phi_{1}(z)+\phi_{1}(\zeta)\right)
$$

then

$D_{1}^{-} U(z, t)=\frac{1}{c_{1}(z)} \int_{D}^{z} d \zeta c_{1}^{\prime}(\zeta) u\left(\zeta, t-\phi_{1}(z)+\phi_{1}(\zeta)\right)+c_{1}(\zeta) D_{1}^{-} u\left(\zeta, t-\phi_{1}(z)+\phi_{1}(\zeta)\right)$

Finally, the bound (64) follows from the identity

$$
\begin{aligned}
D_{1}^{-} \partial_{t} W(z, t) & =D_{1}^{-}\left(\frac{c_{1}}{2}\left(D_{1}^{+}-D_{1}^{-}\right)\right) W(z, t) \\
& =\frac{c_{1}}{2}\left(D_{1}^{-} w(z, t)-D_{1}^{-} D_{1}^{-} W(z, t)\right)+\frac{c_{1}^{\prime}}{2}\left(w(z, t)+D_{1}^{-} W(z, t)\right)
\end{aligned}
$$

2.6. Estimates for the difference of traces. The estimates in this section are central to the proof. Here we will derive estimates for the difference of solutions of the wave equation restricted to two nearby characteristics. We will do this by changing variables from $(z, t)$ to the coordinates corresponding to the characteristics $t=\tau \pm \phi_{i}(z)$. We then apply the trace estimates from the previous section, which control derivatives in these directions.

To this end, let

$$
\varepsilon=\left\|\phi_{2}-\phi_{1}\right\|_{L^{\infty}[0, D]}
$$

For $0 \leq z \leq D$

$$
\left|\phi_{2}(z)-\phi_{1}(z)\right|=\left|\int_{0}^{z} \frac{1}{c_{2}}-\frac{1}{c_{1}}\right| \leq\left\|\frac{1}{c_{1} c_{2}}\right\|_{L^{\infty}} \sqrt{D}\left\|c_{2}-c_{1}\right\|_{L^{2}},
$$

so invoking assumption (12) we obtain

$$
\varepsilon=\left\|\phi_{2}-\phi_{1}\right\|_{L^{\infty}[0, D]} \leq C_{1}\left\|c_{2}-c_{1}\right\|_{L^{2}} .
$$

Now we will show that the difference of the traces of solutions of the wave equation along the two nearby characteristics $t=\phi_{i}(z), i=1,2$, is also small.

Proposition 2.8. Suppose that $u$ is smooth. For $i=1,2$, let

$$
\begin{gathered}
\Lambda_{i}=\sup _{-\varepsilon \leq \tau \leq \varepsilon}\left(\left\|D_{i}^{+} u\left(z, \tau+\phi_{i}(z)\right)\right\|_{L^{2}}+\left\|D_{i}^{+} u\left(z, \tau+\phi_{i}(z)\right)\right\|_{L^{2}}\right) \\
\Pi_{i}=\sup _{-\varepsilon \leq \tau \leq \varepsilon}\left(\left\|D_{i}^{+} D_{i}^{+} u\left(z, \tau+\phi_{i}(z)\right)\right\|_{L^{2}}+\left\|D_{i}^{+} D_{i}^{-} u\left(z, \tau+\phi_{i}(z)\right)\right\|_{L^{2}}+\right. \\
\left.\left\|D_{i}^{-} D_{i}^{+} u\left(z, \tau+\phi_{i}(z)\right)\right\|_{L^{2}}+\left\|D_{i}^{-} D_{i}^{-} u\left(z, \tau+\phi_{i}(z)\right)\right\|_{L^{2}}\right) .
\end{gathered}
$$


Then

$$
\left\|u\left(z, \phi_{2}(z)\right)-u\left(z, \phi_{1}(z)\right)\right\|_{L^{2}[0, D]} \leq C_{i} \Lambda_{i}\left\|c_{2}-c_{1}\right\|_{L^{2}[0, D]}
$$

and

$$
\begin{gathered}
\left\|u\left(z, \phi_{2}(z)\right)-u\left(z, \phi_{1}(z)\right)-\left(\phi_{2}(z)-\phi_{1}(z)\right) \partial_{t} u\left(z, \phi_{1}(z)\right)\right\|_{L^{2}[0, D]} \\
\leq K_{i} \Pi_{i}\left\|c_{2}-c_{1}\right\|_{L^{2}[0, D]}^{2}
\end{gathered}
$$

Proof. We will use the following integral form of Hölder's inequality. For $1 \leq p<\infty$,

$$
\left[\int\left|\int u(z, t) d t\right|^{p} d z\right]^{\frac{1}{p}} \leq \int\left[\int|u(z, t)|^{p} d z\right]^{\frac{1}{p}} d t
$$

For simplicity, we will prove the case where $i=1$; the case $i=2$ is the same, mutatis mutandis. Let $\mu(z)=\phi_{2}(z)-\phi_{1}(z)$. Then

$$
\begin{gathered}
\left\|u\left(z, \phi_{2}(z)\right)-u\left(z, \phi_{1}(z)\right)\right\|_{L^{2}}=\left[\int_{0}^{D} d z\left(\int_{\phi_{1}(z)}^{\mu(z)+\phi_{1}(z)} d t \partial_{t} u(z, t)\right)^{2}\right]^{\frac{1}{2}} \\
=\left[\int_{0}^{D} d z\left(\int_{\phi_{1}(z)}^{\mu(z)+\phi_{1}(z)} d t \frac{c_{1}(z)}{2}\left(D_{1}^{+} u(z, t)-D_{1}^{-} u(z, t)\right)\right)^{2}\right]^{\frac{1}{2}}
\end{gathered}
$$

Now make the change of variable from $t$ to the $\tau$ coordinate defined by the characteristics: $t=\tau+\phi_{1}(z)$. If we do this and apply (68), we obtain

$$
\begin{aligned}
& \left\|u\left(z, \phi_{2}(z)\right)-u\left(z, \phi_{1}(z)\right)\right\|_{L^{2}} \\
& \quad=\left[\int_{0}^{D} d z\left(\int_{0}^{\mu(z)} d \tau \frac{c_{1}(z)}{2}\left(D_{1}^{+} u\left(z, \tau+\phi_{1}(z)\right)-D_{1}^{-} u\left(z, \tau+\phi_{1}(z)\right)\right)\right)^{2}\right]^{\frac{1}{2}} \\
& \quad \leq\left\|c_{1}\right\|_{L^{\infty}}\|\mu\|_{L^{\infty}} \sup _{-\varepsilon \leq \tau \leq \varepsilon}\left(\left\|D_{1}^{+} u\left(z, \tau+\phi_{1}(z)\right)\right\|_{L^{2}}+\left\|D_{1}^{+} u\left(z, \tau+\phi_{1}(z)\right)\right\|_{L^{2}}\right) .
\end{aligned}
$$

Then from (65),

$$
\left\|u\left(z, \phi_{2}(z)\right)-u\left(z, \phi_{1}(z)\right)\right\|_{L^{2}} \leq C_{1} \Lambda_{1}\left\|c_{2}-c_{1}\right\|_{L^{2}} .
$$

The proof of (67) follows exactly the same path, starting with the identity

$$
\partial_{t t}^{2}=\frac{c_{i}^{2}}{4}\left(D_{i}^{+} D_{i}^{+}-D_{i}^{-} D_{i}^{+}-D_{i}^{+} D_{i}^{-}+D_{i}^{-} D_{i}^{-}\right)
$$

which follows from $\partial_{t}=\frac{1}{2} c_{i}\left(D_{i}^{+}-D_{i}^{-}\right)$. We then proceed as above in order to bound

$$
u\left(z, \phi_{2}(z)\right)-u\left(z, \phi_{1}(z)\right)-\left(\phi_{2}(z)-\phi_{1}(z)\right) \partial_{t} u\left(z, \phi_{1}(z)\right)=\int_{\phi_{1}(z)}^{\phi_{2}(z)} d r \int_{\phi_{1}(z)}^{r} d s \partial_{t t}^{2} u(z, s)
$$


Next we apply the previous proposition to the functions considered in Section 2.5. PROPOSITION 2.9. There exist constants $C_{1}$ and $K_{1}$ such that

$$
\begin{aligned}
\left\|w\left(z, \phi_{2}(z)\right)-w\left(z, \phi_{1}(z)\right)\right\|_{L^{2}[0, D]} & \leq C_{1}\left\|c_{2}-c_{1}\right\|_{L^{2}[0, D]}\|f\|_{L^{2}[0, T]} \\
\left\|D_{1}^{-} w\left(z, \phi_{2}(z)\right)-D_{1}^{-} w\left(z, \phi_{1}(z)\right)\right\|_{L^{2}[0, D]} & \leq K_{1}\left\|c_{2}-c_{1}\right\|_{L^{2}[0, D]}\|f\|_{L^{2}[0, T]} \\
\left\|\partial_{t} W\left(z, \phi_{2}(z)\right)-\partial_{t} W\left(z, \phi_{1}(z)\right)\right\|_{L^{2}[0, D]} & \leq C_{1}\left\|c_{2}-c_{1}\right\|_{L^{2}[0, D]}\|f\|_{L^{2}[0, T]}
\end{aligned}
$$

and

$$
\begin{gathered}
\left\|W\left(z, \phi_{2}(z)\right)-W\left(z, \phi_{1}(z)\right)-\left(\phi_{2}(z)-\phi_{1}(z)\right) \partial_{t} W\left(z, \phi_{1}(z)\right)\right\|_{L^{2}[0, D]} \\
\leq K_{1}\left\|c_{2}-c_{1}\right\|_{L^{2}[0, D]}^{2}\|f\|_{L^{2}[0, T]}
\end{gathered}
$$

If $v$ solves

$$
\begin{aligned}
\square_{2} v & =a(z) \partial_{t} w \\
\partial_{z} v(0, t) & =0 \\
v & \equiv 0, \quad t \geq T-\phi_{1}(z)
\end{aligned}
$$

where $a(z)$ is a smooth function, then there exists a constant $K_{2}$ such that

$$
\left\|v\left(z, \phi_{2}(z)\right)-v\left(z, \phi_{1}(z)\right)\right\|_{L^{2}[0, D]} \leq K_{2}\|a\|_{L^{2}[0, D]}\|f\|_{L^{2}[0, T]} .
$$

Proof. We apply Proposition 2.8 and the trace estimates of Section 2.5.

To obtain (69), we use the estimates on $D_{1}^{+} w$ and $D_{1}^{-} w$ in (48) and (50). Estimate (70) follows from (54) and (52), since $D_{1}^{+} D_{1}^{-} w=\left(c_{1}^{\prime} / c_{1}^{2}\right) \partial_{t} w$ (see (32)). Similarly, (71) follows from (61) and (62). The second-order estimate (72) follows from Proposition 2.7 .

Finally (74) follows from (56) and (58).

2.7. A bound on area integrals. Besides estimating the difference of traces along characteristics, we will also need to estimate differences of the form

$$
\int_{0}^{D} d z \int_{\phi_{2}(z)}^{T-\phi_{1}(z)} d t a(z) \partial_{t} w \partial_{t t}^{2} u_{2}-\int_{0}^{D} d z \int_{\phi_{1}(z)}^{T-\phi_{1}(z)} d t a(z) \partial_{t} w \partial_{t t}^{2} u_{1}
$$

Here we will see the first application of the estimates for the difference of traces from Section 2.6. We have the following estimate.

Proposition 2.10. There exists a constant $B_{1}$ such that for any smooth $a=a(z)$,

$$
\begin{gathered}
\left|\int_{0}^{D} d z \int_{\phi_{2}(z)}^{T-\phi_{1}(z)} d t a(z) \partial_{t} w \partial_{t t}^{2} u_{2}-\int_{0}^{D} d z \int_{\phi_{1}(z)}^{T-\phi_{1}(z)} d t a(z) \partial_{t} w \partial_{t t}^{2} u_{1}\right| \\
\leq B_{1}\left\|c_{2}-c_{1}\right\|_{H^{1}}\|a\|_{L^{2}}\|f\|_{L^{2}} .
\end{gathered}
$$


Dmsof. Let $v$ be the solution of

$$
\begin{aligned}
\square_{2} v & =a(z) \partial_{t} w \\
\partial_{z} v(0, t) & =0 \\
v & =0, \quad t \geq T-\phi_{1}(z)
\end{aligned}
$$

Now we apply the Stokes' theorem identity (15) to the two regions

$$
\left\{(z, t) \mid 0 \leq z \leq D, \phi_{i}(z) \leq t \leq 2 \phi_{i}(D)-\phi_{i}(z)\right\}
$$

for $i=1,2$. In the case $i=2$ we have

$$
\int_{0}^{D} d z \int_{\phi_{2}(z)}^{T-\phi_{1}(z)} d t a(z) \partial_{t} w \partial_{t t}^{2} u_{2}=-\int_{0}^{D} d z D_{2}^{+} v\left(z, \phi_{2}(z)\right) D_{2}^{+} u_{2}\left(z, \phi_{2}(z)\right)
$$

On the other hand, since

$$
\square_{2} w=\left(\square_{2}-\square_{1}\right) w=\left(\frac{1}{c_{2}^{2}}-\frac{1}{c_{1}^{2}}\right) \partial_{t t}^{2} w
$$

the Stokes' theorem identity (15) in the case $i=1$ yields

$$
\begin{aligned}
& \int_{0}^{D} d z \int_{\phi_{1}(z)}^{T-\phi_{1}(z)} d t a(z) \partial_{t} w \partial_{t t}^{2} u_{1}=\int_{0}^{D} d z \int_{\phi_{1}(z)}^{T-\phi_{1}(z)} d t\left(\left(\square_{2}-\square_{1}\right)+\square_{1}\right) v \partial_{t t}^{2} u_{1} \\
& \quad=\int_{0}^{D} d z \int_{\phi_{1}(z)}^{T-\phi_{1}(z)} d t\left(\frac{1}{c_{2}^{2}}-\frac{1}{c_{1}^{2}}\right) \partial_{t t}^{2} v \partial_{t t}^{2} u_{1}-\int_{0}^{D} d z D_{1}^{+} v\left(z, \phi_{1}(z)\right) D_{1}^{+} \partial_{t} u_{1}\left(z, \phi_{1}(z)\right) .
\end{aligned}
$$

Recall from the progressing wave expansion that $\partial_{t t}^{2} u_{1}\left(z, \phi_{1}(z)\right)=b_{1}(z)($ see $(22))$. Then we may integrate by parts in $t$ to obtain

$$
\begin{aligned}
& \int_{0}^{D} d z \int_{\phi_{1}(z)}^{T-\phi_{1}(z)} d t\left(\frac{1}{c_{2}^{2}}-\frac{1}{c_{1}^{2}}\right) \partial_{t t}^{2} v \partial_{t t}^{2} u_{1}= \\
& \quad \int_{0}^{D} d z\left(\frac{1}{c_{2}^{2}(z)}-\frac{1}{c_{1}^{2}(z)}\right) \partial_{t} v\left(z, \phi_{1}(z)\right) b_{1}\left(z, \phi_{1}(z)\right) \\
& \quad-\int_{0}^{D} d z \int_{\phi_{1}(z)}^{T-\phi_{1}(z)} d t\left(\frac{1}{c_{2}^{2}}-\frac{1}{c_{1}^{2}}\right) \partial_{t} v \partial_{t t t}^{3} u_{1}
\end{aligned}
$$

From the formula (20) for $b_{1}$ and the trace estimate (59) for $\partial_{t} v\left(z, \phi_{1}(z)\right)$ from Proposition 2.6, we obtain the bound

$$
\left|\int_{0}^{D} d z\left(\frac{1}{c_{2}^{2}(z)}-\frac{1}{c_{1}^{2}(z)}\right) \partial_{t} v\left(z, \phi_{1}(z)\right) b_{1}\left(z, \phi_{1}(z)\right)\right| \leq K_{1}\left\|c_{2}-c_{1}\right\|_{L^{2}}\|a\|_{L^{2}}\|f\|_{L^{2}} .
$$

Meanwhile, from the sideways estimates (42) and (57) we obtain

$$
\left|\int_{0}^{D} d z \int_{\phi_{1}(z)}^{T-\phi_{1}(z)} d t\left(\frac{1}{c_{2}^{2}}-\frac{1}{c_{1}^{2}}\right) \partial_{t} v \partial_{t t t}^{3} u_{1}\right| \leq B_{1}\left\|c_{2}-c_{1}\right\|_{L^{2}}\|a\|_{L^{2}}\|f\|_{L^{2}} .
$$


Thus

$$
\left|\int_{0}^{D} d z \int_{\phi_{1}(z)}^{T-\phi_{1}(z)} d t\left(\frac{1}{c_{2}^{2}}-\frac{1}{c_{1}^{2}}\right) \partial_{t t}^{2} v \partial_{t t}^{2} u_{1}\right| \leq B_{1}\left\|c_{2}-c_{1}\right\|_{L^{2}}\|a\|_{L^{2}}\|f\|_{L^{2}} .
$$

Meanwhile, since $u_{i}\left(z, \phi_{i}(z)\right)=a_{i}(z)$,

$$
\begin{aligned}
\int_{0}^{D} d z & D_{2}^{+} v\left(z, \phi_{2}(z)\right) D_{2}^{+} u_{2}\left(z, \phi_{2}(z)\right)-\int_{0}^{D} d z D_{1}^{+} v\left(z, \phi_{1}(z)\right) D_{1}^{+} u_{1}\left(z, \phi_{1}(z)\right) \\
\quad= & \int_{0}^{D} d z a_{2}^{\prime}(z) D_{2}^{+} v\left(z, \phi_{2}(z)\right)-\int_{0}^{D} d z a_{1}^{\prime}(z) D_{1}^{+} v\left(z, \phi_{1}(z)\right) \\
\quad= & \int_{0}^{D} d z\left(a_{2}-a_{1}\right)^{\prime}(z) D_{2}^{+} v\left(z, \phi_{2}(z)\right)-\int_{0}^{D} d z a_{1}^{\prime \prime}(z)\left[v\left(z, \phi_{2}(z)\right)-v\left(z, \phi_{1}(z)\right)\right]
\end{aligned}
$$

Applying (56) to the first integral and (74) to the second, we obtain

$$
\begin{gathered}
\left|\int_{0}^{D} d z D_{2}^{+} v\left(z, \phi_{2}(z)\right) D_{2}^{+} u_{2}\left(z, \phi_{2}(z)\right)-\int_{0}^{D} d z D_{1}^{+} v\left(z, \phi_{1}(z)\right) D_{1}^{+} u_{1}\left(z, \phi_{1}(z)\right)\right| \\
\leq K_{1}\left\|c_{2}-c_{1}\right\|_{H^{1}}\|a\|_{L^{2}}\|f\|_{L^{2}} .
\end{gathered}
$$

3. The proof of Theorem 1.1: Lipschitz continuity. We will now prove Theorem 1.1. The progressing wave expansion tells us that along the characteristics $t=\phi_{i}(z)$, $i=1,2$,

$$
u_{i}\left(z, \phi_{i}(z)\right)=a_{i}(z)
$$

Thus (30) and (31) become

$$
\int_{0}^{T} d t f(t) F\left(c_{1}\right)=\int_{0}^{D} d z a_{1}^{\prime}(z) D_{1}^{+} w\left(z, \phi_{1}(z)\right)
$$

and

$$
\int_{\mathcal{J}_{0}}^{T} d t f(t) F\left(c_{2}\right)=\int_{0}^{D} d z a_{2}^{\prime}(z) D_{2}^{+} w\left(z, \phi_{2}(z)\right)+\int_{0}^{D} d z \int_{\phi_{2}(z)}^{T-\phi_{1}(z)} d t \partial_{t} u_{2} \square_{2} w
$$

Once again we rely upon the fact that

$$
\square_{2} w=\left(\square_{2}-\square_{1}\right) w=\left(\frac{1}{c_{2}^{2}}-\frac{1}{c_{1}^{2}}\right) \partial_{t t}^{2} w
$$

We use this and integrate by parts in $t$. Bearing in mind that $w \equiv 0$ for $t \geq T-\phi_{1}(z)$ and $\partial_{t} u_{2}\left(z, \phi_{2}(z)\right)=b_{2}(z)$, we obtain

$$
\begin{aligned}
& \int_{0}^{D} d z \int_{\phi_{2}(z)}^{T-\phi_{1}(z)} d t \partial_{t} u_{2} \square_{2} w=\int_{0}^{D} d z \int_{\phi_{2}(z)}^{T-\phi_{1}(z)} d t\left(\frac{1}{c_{2}^{2}}-\frac{1}{c_{1}^{2}}\right) \partial_{t t}^{2} w \partial_{t} u_{2} \\
& \quad=\int_{0}^{D} d z\left(\frac{1}{c_{2}^{2}(z)}-\frac{1}{c_{1}^{2}(z)}\right) b_{2}(z) \partial_{t} w\left(z, \phi_{2}(z)\right)-\int_{0}^{D} d z \int_{\phi_{2}(z)}^{T-\phi_{1}(z)} d t\left(\frac{1}{c_{2}^{2}}-\frac{1}{c_{1}^{2}}\right) \partial_{t} w \partial_{t t}^{2} u_{2}
\end{aligned}
$$


and so

$$
\begin{aligned}
& \int_{0}^{T} d t f(t) F\left(c_{2}\right)=\int_{0}^{D} d z a_{2}^{\prime}(z) D_{2}^{+} w\left(z, \phi_{2}(z)\right) \\
& \quad+\int_{0}^{D} d z\left(\frac{1}{c_{2}^{2}(z)}-\frac{1}{c_{1}^{2}(z)}\right) b_{2}(z) \partial_{t} w\left(z, \phi_{2}(z)\right)-\int_{0}^{D} d z \int_{\phi_{2}(z)}^{T-\phi_{1}(z)} d t\left(\frac{1}{c_{2}^{2}}-\frac{1}{c_{1}^{2}}\right) \partial_{t} w \partial_{t t}^{2} u_{2} .
\end{aligned}
$$

We also have

$$
\begin{aligned}
& \int_{0}^{D} d z a_{2}^{\prime}(z) D_{2}^{+} w\left(z, \phi_{2}(z)\right)-\int_{0}^{D} d z a_{1}^{\prime}(z) D_{1}^{+} w\left(z, \phi_{1}(z)\right)= \\
& \left.\quad \int_{0}^{D} d z\left[a_{2}^{\prime}(z)-a_{1}^{\prime}(z)\right)\right] D_{2}^{+} w\left(z, \phi_{2}(z)\right)-\int_{0}^{D} d z a_{1}^{\prime \prime}(z)\left[w\left(z, \phi_{2}(z)\right)-w\left(z, \phi_{1}(z)\right)\right] .
\end{aligned}
$$

Thus

$$
\begin{aligned}
& \int_{0}^{T} d t f(t)\left[F\left(c_{2}\right)-F\left(c_{1}\right)\right]= \\
& \left.\int_{0}^{D} d z\left[a_{2}^{\prime}(z)-a_{1}^{\prime}(z)\right)\right] D_{2}^{+} w\left(z, \phi_{2}(z)\right)-\int_{0}^{D} d z a_{1}^{\prime \prime}(z)\left[w\left(z, \phi_{2}(z)\right)-w\left(z, \phi_{1}(z)\right)\right] \\
& +\int_{0}^{D} d z\left(\frac{1}{c_{2}^{2}(z)}-\frac{1}{c_{1}^{2}(z)}\right) b_{2}(z) \partial_{t} w\left(z, \phi_{2}(z)\right)-\int_{0}^{D} d z \int_{\phi_{2}(z)}^{T-\phi_{1}(z)} d t\left(\frac{1}{c_{2}^{2}}-\frac{1}{c_{1}^{2}}\right) \partial_{t} w \partial_{t t}^{2} u_{2}
\end{aligned}
$$

We can bound the terms in (76). From Proposition 2.5 we have the following bound on the first term:

$$
\left.\mid \int_{0}^{D} d z\left[a_{2}^{\prime}(z)-a_{1}^{\prime}(z)\right)\right] D_{2}^{+} w\left(z, \phi_{2}(z)\right) \mid \leq C_{1}\left\|c_{2}-c_{1}\right\|_{H^{1}}\|f\|_{L^{2}} .
$$

Using (69), we bound the second term in (76) as

$$
\left|\int_{0}^{D} d z a_{1}^{\prime \prime}(z)\left[w\left(z, \phi_{2}(z)\right)-w\left(z, \phi_{1}(z)\right)\right]\right| \leq K_{1}\left\|c_{2}-c_{1}\right\|_{L^{2}}\|f\|_{L^{2}} .
$$

The third term on the right-hand side of (76) is bounded using the expression for $b_{2}$ in (20) and the trace estimate (54) for $\partial_{t} w$ in Proposition 2.4:

$$
\left|\int_{0}^{D} d z\left(\frac{1}{c_{2}^{2}(z)}-\frac{1}{c_{1}^{2}(z)}\right) b_{2}(z) \partial_{t} w\left(z, \phi_{2}(z)\right)\right| \leq K_{2}\left\|c_{2}-c_{1}\right\|_{L^{2}}\|f\|_{L^{2}} .
$$

Finally, we estimate the area integral by means of the bounds on the sideways energy of $\partial_{t} u_{2}$ and $\partial_{t} w$ given in (42) and (49):

$$
\left|\int_{0}^{D} d z \int_{\phi_{2}(z)}^{T-\phi_{1}(z)} d t\left(\frac{1}{c_{2}^{2}}-\frac{1}{c_{1}^{2}}\right) \partial_{t} w \partial_{t t}^{2} u_{2}\right| \leq K_{2}\left\|c_{2}-c_{1}\right\|_{L^{2}}\|f\|_{L^{2}} .
$$

Since we assume that $\left\|c_{2}-c_{1}\right\|_{H^{2}}<r$, we may replace the constants $K_{2}$ with constants $K_{1}$ in the preceding bounds. We conclude that

$$
\left|\int_{0}^{T} d t f(t)\left[F\left(c_{2}\right)-F\left(c_{1}\right)\right]\right| \leq\left[K_{1}\left\|c_{2}-c_{1}\right\|_{L^{2}}+C_{1}\left\|c_{2}-c_{1}\right\|_{H^{1}}\right]\|f\|_{L^{2}}
$$

for any $f \in C_{0}^{\infty}[0, T]$, from which it follows that

$$
\left\|F\left(c_{2}\right)-F\left(c_{1}\right)\right\|_{L^{2}} \leq\left[K_{1}\left\|c_{2}-c_{1}\right\|_{L^{2}}+C_{1}\left\|c_{2}-c_{1}\right\|_{H^{1}}\right]
$$

This proves Theorem 1.1. 


\section{The proof of Theorem 1.2: Regularity.}

4.1. A formula for $D F\left[c_{1}\right](\delta c)$. We begin with a formula for $D F\left[c_{1}\right](\delta c)$. Proposition 4.1.

$$
\begin{aligned}
\langle f, & \left.D F\left[c_{1}\right](\delta c)\right\rangle_{L^{2}[0, T]}=\int_{0}^{D} d z \delta a^{\prime}(z) D_{1}^{+} w\left(z, \phi_{1}(z)\right) \\
& +\int_{0}^{D} d z\left(-a_{1}^{\prime \prime}(z) \delta \phi(z)+\frac{2 \delta c(z)}{c_{1}^{3}(z)} b_{1}(z)\right) \partial_{t} w\left(z, \phi_{1}(z)\right) \\
& +\int_{0}^{D} d z \int_{\phi_{1}(z)}^{T-\phi_{1}(z)} d t \frac{2 \delta c(z)}{c_{1}^{3}(z)} \partial_{t} w(z, t) \partial_{t t}^{2} u_{1}(z, t)
\end{aligned}
$$

Proof. We will need the following integration by parts identity. Suppose $b$ is a smooth function such that $b(0)=0$. Then we claim that

$$
\int_{0}^{D} d z\left(c_{1}^{\frac{1}{2}} b\right)^{\prime}(z) D_{1}^{+} w\left(z, \phi_{1}(z)\right)=\int_{0}^{D} d z c_{1}^{\frac{1}{2}}(z) b^{\prime}(z) \frac{2}{c_{1}(z)} \partial_{t} w\left(z, \phi_{1}(z)\right)
$$

Identity (78) is proved as follows. From (32) we have

$$
\int_{0}^{D} d z c_{1}^{\frac{1}{2}}(z) b(z)\left[\frac{c_{1}^{\prime}(z)}{c_{1}^{2}(z)} \partial_{t} w\left(z, \phi_{1}(z)\right)-\frac{d}{d z} D_{1}^{-} w\left(z, \phi_{1}(z)\right)\right]=0 .
$$

Integrating the second term produces

$$
\begin{aligned}
& -\int_{0}^{D} d z c_{1}^{\frac{1}{2}}(z) b(z) \frac{d}{d z} D_{1}^{-} w\left(z, \phi_{1}(z)\right)= \\
& \quad \int_{0}^{D} d z\left(\frac{1}{2} \frac{c_{1}^{\prime}(z)}{c_{1}^{2}(z)} b(z)+c_{1}^{\frac{1}{2}}(z) b^{\prime}(z)\right) D_{1}^{-} w\left(z, \phi_{1}(z)\right) .
\end{aligned}
$$

Thus,

$$
\begin{aligned}
& \int_{0}^{D} d z c_{1}^{\frac{1}{2}}(z) b(z)\left[\frac{c_{1}^{\prime}(z)}{c_{1}^{2}(z)} \partial_{t} w\left(z, \phi_{1}(z)\right)-\frac{d}{d z} D_{1}^{-} w\left(z, \phi_{1}(z)\right)\right]= \\
& \int_{0}^{D} d z\left(c_{1}^{\frac{1}{2}}\right)^{\prime}(z) b(z) D_{1}^{+} w\left(z, \phi_{1}(z)\right)+\int_{0}^{D} d z c_{1}^{\frac{1}{2}}(z) b^{\prime}(z) D_{1}^{-} w\left(z, \phi_{1}(z)\right)=0
\end{aligned}
$$

and so

$$
\begin{gathered}
\int_{0}^{D} d z\left(c_{1}^{\frac{1}{2}}\right)^{\prime}(z) b(z) D_{1}^{+} w\left(z, \phi_{1}(z)\right)+\int_{0}^{D} d z c_{1}^{\frac{1}{2}}(z) b^{\prime}(z) D_{1}^{+} w\left(z, \phi_{1}(z)\right) \\
-\int_{0}^{D} d z c_{1}^{\frac{1}{2}}(z) b^{\prime}(z) \frac{2}{c_{1}(z)} \partial_{t} w\left(z, \phi_{1}(z)\right)=0
\end{gathered}
$$

which is (78).

We now turn to deriving (77). Applying the Stokes' theorem identity (15) and a straightforward limiting argument to handle the singular nature of the integrand, we 
obtain

$$
\begin{gathered}
\int_{0}^{T} d t f(t) \partial_{t} \delta u(0, t)=\int_{0}^{D} d z\left(\delta a-b_{1} \delta \phi\right)^{\prime}(z) D_{1}^{+} w\left(z, \phi_{1}(z)\right) \\
\quad+\int_{0}^{D} d z \int_{\phi_{1}(z)}^{T-\phi_{1}(z)} d t \frac{2 \delta c(z)}{c_{1}^{3}(z)} \partial_{t} w(z, t) \partial_{t t}^{2} u_{1}(z, t)
\end{gathered}
$$

We then apply (78) to obtain the following:

$$
\int_{0}^{D} d z\left(b_{1} \delta \phi\right)^{\prime}(z) D_{1}^{+} w\left(z, \phi_{1}(z)\right)=\int_{0}^{D} d z c_{1}^{\frac{1}{2}}(z)\left(c_{1}^{-\frac{1}{2}} b_{1} \delta \phi\right)^{\prime}(z) \frac{2}{c_{1}(z)} \partial_{t} w\left(z, \phi_{1}(z)\right) .
$$

However, we see from the formula $(20)$ for $b_{1}$ that

$$
\left(c_{1}^{-\frac{1}{2}} b_{1}\right)^{\prime}(z)=\frac{1}{2} c_{1}^{-\frac{1}{2}}(z) a_{1}^{\prime \prime}(z)
$$

so

$$
c_{1}^{\frac{1}{2}}(z)\left(c_{1}^{-\frac{1}{2}} b_{1} \delta \phi\right)^{\prime}(z)=c_{1}^{\frac{1}{2}}(z)\left(c_{1}^{-\frac{1}{2}}(z) a_{1}^{\prime \prime}(z) \delta \phi(z)-c_{1}^{-\frac{1}{2}}(z) \frac{\delta c(z)}{c_{1}^{2}(z)}\right) b_{1}(z)
$$

Thus

$$
\int_{0}^{D} d z\left(b_{1} \delta \phi\right)^{\prime}(z) D_{1}^{+} w\left(z, \phi_{1}(z)\right)=\int_{0}^{D} d z\left(a_{1}^{\prime \prime}(z) \delta \phi(z)-\frac{2 \delta c(z)}{c_{1}^{3}(z)} b_{1}(z)\right) \partial_{t} w\left(z, \phi_{1}(z)\right)
$$

Proposition 4.1 then follows from this latter equality and (79).

4.2. Continuity of $D F$. Proposition 4.1 shows that $D F[c]: H^{1} \rightarrow L^{2}$ for $c \in H^{2}$, since we can easily use the trace estimates in Proposition 2.4 to derive a bound

$$
\left|\langle f, D F[c](\delta c)\rangle_{L^{2}[0, T]}\right| \leq K\|\delta c\|_{H^{1}}\|f\|_{L^{2}}
$$

for all $f \in C_{0}^{\infty}[0, T]$, where $K=K\left(\|c\|_{H^{2}[0, D]}, c_{m i n}, D\right)$. However, in order to show that $D F[c]$ depends continuously on $c$, we must require more smoothness of both the velocity and the admissible perturbations $\delta c$. The following is part (ii) of Theorem 1.2.

Proposition 4.2. There exists a constant $B_{1}$ such that if $\left\|c_{2}-c_{1}\right\|_{H^{2}[0, D]}<r$, then

$$
\left\|D F\left[c_{2}\right]-D F\left[c_{1}\right]\right\| \leq B_{1}\left\|c_{2}-c_{1}\right\|_{H^{2}[0, D]}
$$

where the operator norm is taken over operators from $H^{2}[0, D]$ to $L^{2}[0, T]$.

Proof. We begin with the identity (79) from the proof of Proposition 4.1: For $i=1,2$,

$$
\begin{gathered}
\int_{0}^{T} d t f(t) \partial_{t} \delta u_{i}(0, t)=\int_{0}^{D} d z\left(\delta a_{i}-b_{i} \delta \phi_{i}\right)^{\prime}(z) D_{i}^{+} w\left(z, \phi_{i}(z)\right) \\
+\int_{0}^{D} d z \int_{\phi_{i}(z)}^{T-\phi_{1}(z)} d t \frac{2 \delta c(z)}{c_{i}^{3}(z)} \partial_{t} w(z, t) \partial_{t t}^{2} u_{i}(z, t) .
\end{gathered}
$$


Then

$$
\begin{aligned}
& \left\langle f,\left(D F\left[c_{2}\right]-D F\left[c_{1}\right]\right)(\delta c)\right\rangle_{L^{2}[0, T]}= \\
& \quad \int_{0}^{D} d z\left(\delta a_{2}-b_{2} \delta \phi_{2}\right)^{\prime}(z) D_{2}^{+} w\left(z, \phi_{2}(z)\right)-\int_{0}^{D} d z\left(\delta a_{1}-b_{1} \delta \phi_{1}\right)^{\prime}(z) D_{1}^{+} w\left(z, \phi_{1}(z)\right) \\
& \quad+\int_{0}^{D} d z \int_{\phi_{2}(z)}^{T-\phi_{1}(z)} d t \frac{2 \delta c}{c_{2}^{3}} \partial_{t} w \partial_{t t}^{2} u_{2}-\int_{0}^{D} d z \int_{\phi_{1}(z)}^{T-\phi_{1}(z)} d t \frac{2 \delta c}{c_{1}^{3}} \partial_{t} w \partial_{t t}^{2} u_{1} .
\end{aligned}
$$

Aftor adding and subtracting a term to the first difference we can integrate to obtain

$$
\begin{aligned}
\int_{0}^{D} d z(\delta & \left.a_{2}-b_{2} \delta \phi_{2}\right)^{\prime}(z) D_{2}^{+} w\left(z, \phi_{2}(z)\right)-\int_{0}^{D} d z\left(\delta a_{1}-b_{1} \delta \phi_{1}\right)^{\prime}(z) D_{1}^{+} w\left(z, \phi_{1}(z)\right) \\
= & \int_{0}^{D} d z\left[\left(\delta a_{2}-b_{2} \delta \phi_{2}\right)^{\prime}(z)-\left(\delta a_{1}-b_{1} \delta \phi_{1}\right)^{\prime}(z)\right] D_{1}^{+} w\left(z, \phi_{1}(z)\right) \\
& -\int_{0}^{D} d z\left(\delta a_{1}-b_{1} \delta \phi_{1}\right)^{\prime \prime}(z)\left[w\left(z, \phi_{2}(z)\right)-w\left(z, \phi_{1}(z)\right)\right] .
\end{aligned}
$$

Applying the trace estimates in Propositions 2.4 and 2.9 we can bound the preceding:

$$
\begin{gathered}
\left|\int_{0}^{D} d z\left(\delta a_{2}-b_{2} \delta \phi_{2}\right)^{\prime}(z) D_{2}^{+} w\left(z, \phi_{2}(z)\right)-\int_{0}^{D} d z\left(\delta a_{1}-b_{1} \delta \phi_{1}\right)^{\prime}(z) D_{1}^{+} w\left(z, \phi_{1}(z)\right)\right| \\
\leq K_{1}\left\|c_{2}-c_{1}\right\|_{H^{2}}\|\delta c\|_{H^{2}}\|f\|_{L^{2}} .
\end{gathered}
$$

Meanwhile, from Proposition 2.10 we obtain the estimate

$$
\begin{aligned}
& \left|\int_{0}^{D} d z \int_{\phi_{2}(z)}^{T-\phi_{1}(z)} d t \frac{2 \delta c}{c_{2}^{3}} \partial_{t} w \partial_{t t}^{2} u_{2}-\int_{0}^{D} d z \int_{\phi_{1}(z)}^{T-\phi_{1}(z)} d t \frac{2 \delta c}{c_{1}^{3}} \partial_{t} w \partial_{t t}^{2} u_{1}\right| \\
& \leq\left|\int_{0}^{D} d z \int_{\phi_{2}(z)}^{T-\phi_{1}(z)} d t \frac{2 \delta c}{c_{2}^{3}} \partial_{t} w \partial_{t t}^{2} u_{2}-\int_{0}^{D} d z \int_{\phi_{1}(z)}^{T-\phi_{1}(z)} d t \frac{2 \delta c}{c_{2}^{3}} \partial_{t} w \partial_{t t}^{2} u_{1}\right| \\
& \quad+\left|\int_{0}^{D} d z \int_{\phi_{1}(z)}^{T-\phi_{1}(z)} d t\left(\frac{2 \delta c}{c_{2}^{3}}-\frac{2 \delta c}{c_{1}^{3}}\right) \partial_{t} w \partial_{t t}^{2} u_{1}\right| \\
& \leq B_{1}\left\|c_{2}-c_{1}\right\|_{L^{2}}\|\delta c\|_{L^{2}}\|f\|_{L^{2}} .
\end{aligned}
$$

Thus we conclude that

$$
\left|\left\langle f,\left(D F\left[c_{2}\right]-D F\left[c_{1}\right]\right)(\delta c)\right\rangle_{L^{2}[0, T]}\right| \leq B_{1}\left\|c_{2}-c_{1}\right\|_{H^{2}}\|\delta c\|_{H^{2}}\|f\|_{L^{2}}
$$

for all $f \in C_{0}^{\infty}[0, T]$, and therefore

$$
\left\|D F\left[c_{2}\right]-D F\left[c_{1}\right]\right\| \leq B_{1}\left\|c_{2}-c_{1}\right\|_{H^{2}} .
$$


4.3. Estimating the linearization error. From (76) and (77) we obtain

$$
\begin{aligned}
\langle f, & \left.F\left(c_{2}\right)-F\left(c_{1}\right)-D F\left[c_{1}\right](\delta c)\right\rangle_{L^{2}[0, T]}= \\
& \int_{0}^{D} d z\left(a_{2}-a_{1}-\delta a\right)^{\prime}(z) D_{2}^{+} w\left(z, \phi_{2}(z)\right) \\
& +\int_{0}^{D} d z \delta a^{\prime}(z)\left[D_{2}^{+} w\left(z, \phi_{2}(z)\right)-D_{1}^{+} w\left(z, \phi_{1}(z)\right)\right] \\
& -\int_{0}^{D} d z a_{1}^{\prime \prime}(z)\left[w\left(z, \phi_{2}(z)\right)-w\left(z, \phi_{1}(z)\right)-\left(\phi_{2}(z)-\phi_{1}(z)\right) \partial_{t} w\left(z, \phi_{1}(z)\right)\right] \\
& -\int_{0}^{D} d z a_{1}^{\prime \prime}(z)\left[\phi_{2}(z)-\phi_{1}(z)-\delta \phi(z)\right] \partial_{t} w\left(z, \phi_{1}(z)\right) \\
& -\int_{0}^{D} d z\left(\frac{1}{c_{2}^{2}(z)}-\frac{1}{c_{1}^{2}(z)}+\frac{2 \delta c(z)}{c_{1}^{3}(z)}\right) b_{2}(z) \partial_{t} w\left(z, \phi_{2}(z)\right) \\
& +\int_{0}^{D} d z \frac{2 \delta c(z)}{c_{1}^{3}(z)}\left(b_{2}(z)-b_{1}(z)\right) \partial_{t} w\left(z, \phi_{2}(z)\right) \\
& +\int_{0}^{D} d z \frac{2 \delta c(z)}{c_{1}^{3}(z)} b_{1}(z)\left[\partial_{t} w\left(z, \phi_{2}(z)\right)-\partial_{t} w\left(z, \phi_{1}(z)\right)\right] \\
& -\int_{0}^{D} d z \int_{\phi_{2}(z)}^{T-\phi_{1}(z)} d t\left(\frac{1}{c_{2}^{2}}-\frac{1}{c_{1}^{2}}+\frac{2 \delta c}{c_{1}^{3}}\right) \partial_{t} w \partial_{t t}^{2} u_{2} \\
& +\int_{0}^{D} d z \int_{\phi_{2}(z)}^{T-\phi_{1}(z)} d t \frac{2 \delta c}{c_{1}^{3}} \partial_{t} w \partial_{t t}^{2} u_{2}-\int_{0}^{D} d z \int_{\phi_{1}(z)}^{T-\phi_{1}(z)} d t \frac{2 \delta c}{c_{1}^{3}} \partial_{t} w \partial_{t t}^{2} u_{1}
\end{aligned}
$$

Estimates for most of these terms are immediate. From (27) and (53),

$$
\left|\int_{0}^{D} d z\left(a_{2}-a_{1}-\delta a\right)^{\prime}(z) D_{2}^{+} w\left(z, \phi_{2}(z)\right)\right| \leq C_{1}\left\|c_{2}-c_{1}\right\| L_{L^{2}}\left\|c_{2}-c_{1}\right\|_{H^{2}}\|f\|_{L^{2}} .
$$

Integration by parts and the trace difference estimate (69) yields

$$
\left|\int_{0}^{D} d z \delta a^{\prime}(z)\left[D_{2}^{+} w\left(z, \phi_{2}(z)\right)-D_{1}^{+} w\left(z, \phi_{1}(z)\right)\right]\right| \leq K_{1}\left\|c_{2}-c_{1}\right\|_{L^{2}}\left\|c_{2}-c_{1}\right\|_{H^{1}}\|f\|_{L^{2}} .
$$

From the trace estimate (51),

$$
\left|\int_{0}^{D} d z a_{1}^{\prime \prime}(z)\left[\phi_{2}(z)-\phi_{1}(z)-\delta \phi(z)\right] \partial_{t} w\left(z, \phi_{1}(z)\right)\right| \leq K_{1}\left\|c_{2}-c_{1}\right\|_{L^{2}}^{2}\|f\|_{L^{2}} .
$$

The next term in (81) is bounded using the definition of $b_{2}$ in (20) and (54):

$$
\left|\int_{J_{0}}^{D} d z\left(\frac{1}{c_{2}^{2}(z)}-\frac{1}{c_{1}^{2}(z)}+\frac{2 \delta c(z)}{c_{1}^{3}(z)}\right) b_{2}(z) \partial_{t} w\left(z, \phi_{2}(z)\right)\right| \leq K_{1}\left\|c_{2}-c_{1}\right\|_{L^{2}}^{2}\|f\|_{L^{2}} .
$$

Using (26) and (54), we have

$$
\left|\int_{0}^{D} d z \frac{2 \delta c(z)}{c_{1}^{3}(z)}\left(b_{2}(z)-b_{1}(z)\right) \partial_{t} w\left(z, \phi_{2}(z)\right)\right| \leq K_{1}\left\|c_{2}-c_{1}\right\|_{L^{2}}\left\|c_{2}-c_{1}\right\|_{H^{2}}\|f\|_{L^{2}} .
$$


Finally, from (41) and (49), which bound the energy of $\partial_{t} u_{2}$ and $w$ on vertical strips, we have

$$
\left|\int_{0}^{D} d z \int_{\phi_{2}(z)}^{T-\phi_{1}(z)} d t\left(\frac{1}{c_{2}^{2}}-\frac{1}{c_{1}^{2}}+\frac{2 \delta c}{c_{1}^{3}}\right) \partial_{t} w \partial_{t t}^{2} u_{2}\right| \leq K_{1}\left\|c_{2}-c_{1}\right\|_{L^{2}}^{2}\|f\|_{L^{2}} .
$$

Three terms remain. The first of these is

$$
\int_{0}^{D} d z a_{1}^{\prime \prime}(z)\left[w\left(z, \phi_{2}(z)\right)-w\left(z, \phi_{1}(z)\right)-\left(\phi_{2}(z)-\phi_{1}(z)\right) \partial_{t} w\left(z, \phi_{1}(z)\right)\right]
$$

Recall the function $W(z, t)$ from (60) and note that

$$
\begin{aligned}
\frac{d}{d z} W\left(z, \phi_{1}(z)\right) & =w(z, t) \\
\frac{d}{d z} W\left(z, \phi_{2}(z)\right) & =\left(\phi_{2}-\phi_{1}\right)^{\prime}(z) \partial_{t} W\left(z, \phi_{2}(z)\right) .
\end{aligned}
$$

This enables us to integrate (81) by parts:

$$
\begin{aligned}
\int_{0}^{D} d z & a_{1}^{\prime \prime}(z)\left[w\left(z, \phi_{2}(z)\right)-w\left(z, \phi_{1}(z)\right)-\left(\phi_{2}(z)-\phi_{1}(z)\right) \partial_{t} w\left(z, \phi_{1}(z)\right)\right] \\
= & \int_{0}^{D} d z a_{1}^{\prime \prime}(z)\left[\frac{d}{d z}\left(W\left(z, \phi_{2}(z)\right)\right)-\left(\phi_{2}-\phi_{1}\right)^{\prime}(z) \partial_{t} W\left(z, \phi_{2}(z)\right)\right] \\
& -\int_{0}^{D} d z a_{1}^{\prime \prime}(z) \frac{d}{d z}\left(W\left(z, \phi_{1}(z)\right)\right)-\int_{0}^{D} d z a_{1}^{\prime \prime}(z) \delta \phi(z) \frac{d}{d z}\left(\partial_{t} W\left(z, \phi_{1}(z)\right)\right) \\
= & -\int_{0}^{D} d z a_{1}^{\prime \prime \prime}(z)\left[W\left(z, \phi_{2}(z)\right)-W\left(z, \phi_{1}(z)\right)-\left(\phi_{2}(z)-\phi_{1}(z)\right) \partial_{t} W\left(z, \phi_{1}(z)\right)\right] \\
& +\int_{0}^{D} d z a_{1}^{\prime \prime}(z)\left(\phi_{2}-\phi_{1}-\delta \phi\right)(z) \partial_{t} W\left(z, \phi_{1}(z)\right) \\
& -\int_{0}^{D} d z a_{1}^{\prime \prime}(z)\left(\phi_{2}-\phi_{1}-\delta \phi\right)^{\prime}(z) \partial_{t} W\left(z, \phi_{2}(z)\right) \\
& +\int_{0}^{D} d z a_{1}^{\prime \prime}(z) \delta \phi^{\prime}(z)\left[\partial_{t} W\left(z, \phi_{2}(z)\right)-\partial_{t} W\left(z, \phi_{1}(z)\right)\right] .
\end{aligned}
$$

We can estimate each term in this latter expression. By the second-order trace difference estinnate (72),

$$
\begin{gathered}
\left|\int_{0}^{D} d z a_{1}^{\prime \prime \prime}(z)\left[W\left(z, \phi_{2}(z)\right)-W\left(z, \phi_{1}(z)\right)-\left(\phi_{2}(z)-\phi_{1}(z)\right) \partial_{t} W\left(z, \phi_{1}(z)\right)\right]\right| \\
\leq K_{1}\left\|\left(c_{1}^{\frac{1}{2}}\right)^{\prime \prime \prime}\right\|_{L^{2}}\left\|c_{2}-c_{1}\right\|_{L^{2}}^{2}\|f\|_{L^{2}} .
\end{gathered}
$$

Next, the definition of $W$ and the bound on $\partial_{t} w\left(z, \phi_{1}(z)\right)$ in Proposition 2.4 enable us to bound $\partial_{t} W\left(z, \phi_{1}(z)\right)$, whence

$$
\left|\int_{0}^{D} d z a_{1}^{\prime \prime}(z)\left(\phi_{2}-\phi_{1}-\delta \phi\right)^{\prime}(z) \partial_{t} W\left(z, \phi_{2}(z)\right)\right| \leq K_{1}\left\|c_{2}-c_{1}\right\|_{L^{2}}^{2}\|f\|_{L^{2}} .
$$


R. M. Lewis and W. W. Symes - Solutions of the wave equation

Finally, from (71) we obtain

$$
\left|\int_{0}^{D} d z a_{1}^{\prime \prime}(z) \delta \phi^{\prime}(z)\left[\partial_{t} W\left(z, \phi_{2}(z)\right)-\partial_{t} W\left(z, \phi_{1}(z)\right)\right]\right| \leq K_{1}\left\|c_{2}-c_{1}\right\|_{L^{2}}^{2}\|f\|_{L^{2}} .
$$

Thus,

$$
\begin{gathered}
\left|\int_{0}^{D} d z a_{1}^{\prime \prime}(z)\left[w\left(z, \phi_{2}(z)\right)-w\left(z, \phi_{1}(z)\right)-\delta \phi(z) \partial_{t} w\left(z, \phi_{1}(z)\right)\right]\right| \\
\leq B_{1}\left\|c_{2}-c_{1}\right\|_{L^{2}}^{2}\|f\|_{L^{2}} .
\end{gathered}
$$

Next we will estimate

$$
\int_{0}^{D} d z \frac{2 \delta c(z)}{c_{1}^{3}(z)} b_{1}(z)\left[\partial_{t} w\left(z, \phi_{2}(z)\right)-\partial_{t} w\left(z, \phi_{1}(z)\right)\right] .
$$

This is straightforward. Since

$$
\partial_{t}=\frac{c_{1}}{2}\left(D_{1}^{+}-D_{1}^{-}\right)=\frac{c_{1} c_{2}}{c_{1}+c_{2}}\left(D_{2}^{+}-D_{1}^{-}\right),
$$

we have

$$
\begin{aligned}
& \int_{0}^{D} d z \frac{2 \delta c(z)}{c_{1}^{3}(z)} b_{1}(z)\left[\partial_{t} w\left(z, \phi_{2}(z)\right)-\partial_{t} w\left(z, \phi_{1}(z)\right)\right] \\
& \quad \int_{0}^{D} d z \frac{2 \delta c(z)}{c_{1}^{3}(z)} b_{1}(z) \frac{c_{1}(z)}{2}\left[D_{2}^{+} w\left(z, \phi_{2}(z)\right)-D_{1}^{+} w\left(z, \phi_{1}(z)\right)\right] \\
& \quad+\int_{0}^{D} d z \frac{2 \delta c(z)}{c_{1}^{3}(z)} b_{1}(z)\left(\frac{c_{1}(z) c_{2}(z)}{c_{1}(z)+c_{2}(z)}-\frac{c_{1}(z)}{2}\right) D_{2}^{+} w\left(z, \phi_{2}(z)\right) \\
& \quad-\int_{0}^{D} d z \frac{2 \delta c(z)}{c_{1}^{3}(z)} b_{1}(z) \frac{c_{1}(z)}{2}\left[D_{1}^{-} w\left(z, \phi_{2}(z)\right)-D_{1}^{-} w\left(z, \phi_{1}(z)\right)\right] \\
& \quad-\int_{0}^{D} d z \frac{2 \delta c(z)}{c_{1}^{3}(z)} b_{1}(z)\left(\frac{c_{1}(z) c_{2}(z)}{c_{1}(z)+c_{2}(z)}-\frac{c_{1}(z)}{2}\right) D_{1}^{-} w\left(z, \phi_{2}(z)\right) .
\end{aligned}
$$

For the first term in this latter identity we integrate by parts and apply (69). The third term we bound using (70); the other two terms are bounded by the trace estimates in Propositions 2.4 and 2.5. We arrive at

$$
\begin{gathered}
\left|\int_{0}^{D} d z \frac{2 \delta c(z)}{c_{1}^{3}(z)} b_{1}(z)\left[\partial_{t} w\left(z, \phi_{2}(z)\right)-\partial_{t} w\left(z, \phi_{1}(z)\right)\right]\right| \\
\leq K_{1}\left\|c_{2}-c_{1}\right\|_{L^{2}}\left\|c_{2}-c_{1}\right\|_{H^{1}}\|f\|_{L^{2}} .
\end{gathered}
$$

Finally, applying Proposition 2.10 to the difference of the area integrals we obtain the bound

$$
\begin{gathered}
\left|\int_{0}^{D} d z \int_{\phi_{2}(z)}^{T-\phi_{1}(z)} d t \frac{2 \delta c}{c_{1}^{3}} \partial_{t} w \partial_{t t}^{2} u_{2}-\int_{0}^{D} d z \int_{\phi_{1}(z)}^{T-\phi_{1}(z)} d t \frac{2 \delta c}{c_{1}^{3}} \partial_{t} w \partial_{t t}^{2} u_{1}\right| \\
\leq B_{1}\left\|c_{2}-c_{1}\right\|_{L^{2}}^{2}\|f\|_{L^{2}} .
\end{gathered}
$$


The conclusion is that

$$
\begin{gathered}
\left|\left\langle f, F\left(c_{2}\right)-F\left(c_{1}\right)-D F\left[c_{1}\right](\delta c)\right\rangle_{L^{2}[0, T]}\right| \\
\leq\left[B_{1}\left\|c_{2}-c_{1}\right\|_{L^{2}}^{2}+K_{1}\left\|c_{2}-c_{1}\right\|_{L^{2}}\left\|c_{2}-c_{1}\right\|_{H^{2}}\right]\|f\|_{L^{2}}
\end{gathered}
$$

for all $f \in C_{0}^{\infty}[0, T]$. This estimate, the continuity of $F$, and the continuity of $D F$ yield Theorem 1.2.

\section{REFERENCES}

[1] A. Bamberger, G. Chavent, and P. Lailly, About the stability of the inverse problem in 1-d wave equations - application to the interpretation of seismic profiles, Applied Mathematics and Optimization, 5 (1979), pp. 1-47.

[2] R. Courant and D. Hilbert, Methods of Mathematical Physics, vol. II, Wiley-Interscience, New York, 1962.

[3] W. W. Symes, Stability properties for the velocity inversion problem, in Mathematical and Computational Methods in Seismic Exploration and Reservoir Modeling, 1985, pp. 128-157. Proceedings of the 1985 SEG/SIAM/SPE meeting in Houston, Texas.

[4] - On the relation between coefficient and boundary values for solutions of Webster's horn equation, SIAM Journal of Mathematical Analysis, 17 (1986), pp. 1400-1420. 